\title{
Surface configurations and wave patterns of thermocapillary convection onboard the SJ10 satellite $\odot$
}

Cite as: Phys. Fluids 31, 044105 (2019); https://doi.org/10.1063/1.5090466

Submitted: 28 January 2019 . Accepted: 19 March 2019 . Published Online: 15 April 2019

Qi Kang (康琦) (D), Di Wu (吴笛) (D), Li Duan (段俐) (D), Jianwu He (贺建武) (D), Liang Hu (胡良) (D), Longsheng Duan (段隆盛) (D), and Wenrui Hu (胡文瑞)

COLLECTIONS

F This paper was selected as Featured
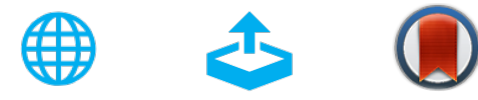

View Online

\section{ARTICLES YOU MAY BE INTERESTED IN}

Effects of shear intensity and aspect ratio on three-dimensional wake characteristics of flow past surface mounted circular cylinder

Physics of Fluids 31, 043602 (2019); https://doi.org/10.1063/1.5081795

Dynamic mode decomposition for the inspection of three-regime separated transitional boundary layers using a least squares method

Physics of Fluids 31, 044103 (2019); https://doi.org/10.1063/1.5086225

Radial planetary vorticity tilting in the leading-edge vortex of revolving wings

Physics of Fluids 31, 041902 (2019); https://doi.org/10.1063/1.5084967

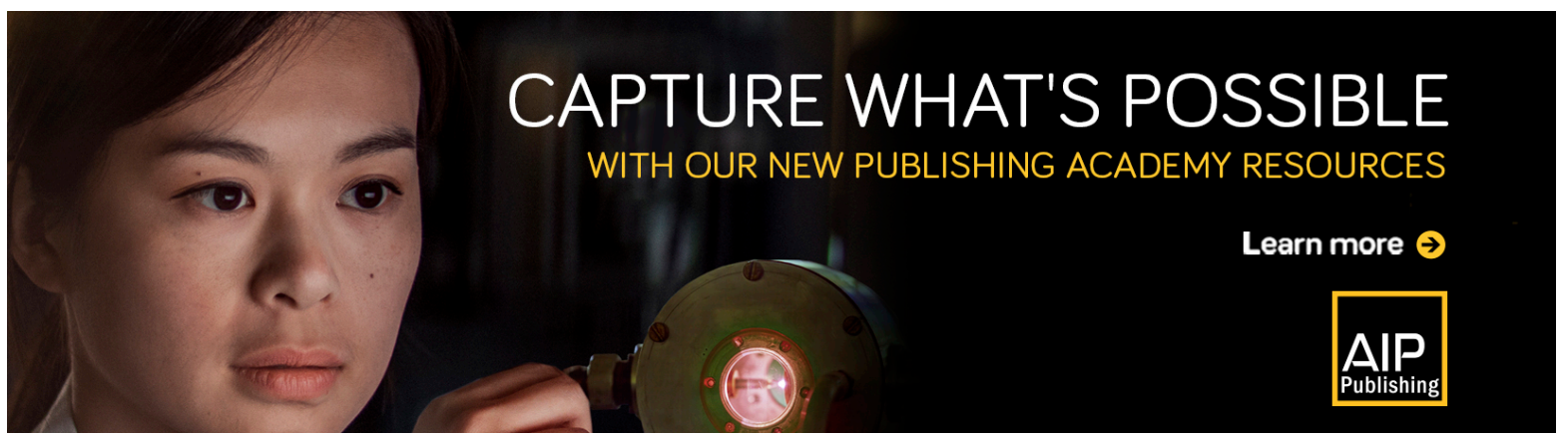




\title{
Surface configurations and wave patterns of thermocapillary convection onboard the SJ10 satellite
}

\author{
Cite as: Phys. Fluids 31, 044105 (2019); doi: 10.1063/1.5090466 \\ Submitted: 28 January 2019 - Accepted: 19 March 2019 • \\ Published Online: 15 April 2019
}

\begin{abstract}
Qi Kang (康琦), 1,2,a) (D Di Wu (吴笛), ${ }^{1, a)}$ (D) Li Duan (段俐), ${ }^{1,2, b)}$ (D) Jianwu He (贺建武), (D) Liang Hu (胡良), Longsheng Duan (段隆盛), (D) and Wenrui Hu (胡文瑞) ${ }^{1,2}$
\end{abstract}

\author{
AFFILIATIONS \\ ${ }^{1}$ Key Laboratory of Microgravity, Institute of Mechanics, Chinese Academy of Sciences, Beijing 100190, \\ People's Republic of China \\ ${ }^{2}$ School of Engineering Sciences, University of Chinese Academy of Sciences, Beijing 100049, People's Republic of China \\ a) Q. Kang and D. Wu are co-first authors. \\ b) Author to whom correspondence should be addressed: duanli@imech.ac.cn
}

\begin{abstract}
A fluid experiment was conducted on China's SJ10 satellite to assess the instability of thermocapillary convection under microgravity. Oscillations of thermocapillary convection in an annular pool were investigated when the difference in temperature exceeded a certain threshold. Dynamics and transitions of the wave pattern were observed using an infrared camera. This work is concerned with the effect of the surface configuration on the wave patterns. There are 3 types of surface configurations that depend on the volume ratio: $V r<0.77$ (zone 1 ), $0.77<V r$ $<1$ (zone 2), and $V r>1$ (zone 3). An irregular local wave occurs at $V r<0.65$, and a regular azimuthal wave occurs at $V r>0.65$. We observed the regular wave with the wavenumber $m=3$ in zone 1 and zone 3 and the competition between $m=3$ and $m=4$ in zone 2 . The nonlinear dynamics of traveling waves, standing waves, and counterpropagating waves were studied. The standing wave appears near the onset and transitions to the traveling wave. With a similar mechanism, counterpropagating waves with $m=3$ and $m=4$ occur in some cases at the onset. Additionally, counterpropagating waves also act as a transitional mode when the traveling wave transitions from $m=4$ to $m=3$. The traveling wave is very stable, but it develops into standing waves or Benjamin-Feir instability under highly supercritical conditions.
\end{abstract}

Published under license by AIP Publishing. https://doi.org/10.1063/1.5090466

\section{INTRODUCTION}

Thermocapillary convection, also called Marangoni convection, is generated by the surface tension gradient due to a difference in temperature. According to the direction of temperature gradient, there are two categories of thermocapillary systems with the gradient parallel or perpendicular to the surface. Thermocapillary convections in these two systems are different, and they form a stationary or oscillatory pattern due to nonlinear effects. Bénard convection with a vertical gradient of temperature forms stationary hexagonal cells. The self-organization of Bénard cells is a canonical subject in pattern formation for a non-equilibrium system, while diffusion-reaction is an example of the oscillatory pattern. However, thermocapillary convection $^{2,3}$ in an open annular or rectangle pool also shows a wave-like pattern. Such oscillatory convections are correlated with dot-like defects or strip defects in crystal growth, ${ }^{4,5}$ so the oscillation is widely studied in the fluid dynamics, materials science, and heat transfer.

Bénard-Marangoni convection shows hexagonal convective cells when heating a thin layer from the bottom. Rayleigh ${ }^{6}$ studied this problem using linear stability analysis and ascribed the convection to buoyancy-driven instability. However, the Rayleigh-Bénard convection occurs in a layer without free surfaces, and the pattern formation is a roll-like convective structure. Pearson ${ }^{7}$ considered the thermocapillary force in his linear stability analysis and explained the original Bénard experiment. The onset condition is validated in the experiments by Koschmieder et al. ${ }^{8}$ Recently, new patterns have been found. Nitschke observed secondary instability such that the hexagon pattern transforms into a square pattern. Nepomnyashchy and Simanovskii ${ }^{10}$ investigated oscillation in two layer systems using 
both linear stability analysis and numerical simulation. Kang et al. ${ }^{1}$ observed the oscillation pattern with particle image velocimetry in the two-layer system, while the oscillatory pattern was not expected in the one-layer system.

In a one-layer system with the temperature gradient parallel to a free surface, an oscillatory pattern appears when the gradient is beyond a threshold. Hydrothermal wave (HTW) theory can explain the oscillation in thermocapillary convection. Instability of hydrothermal waves and convective rolls were proposed by Smith and Davis ${ }^{12}$ when they were performing linear stability analysis to study the thermocapillary convection in an infinite layer. Burguete et al. ${ }^{13}$ found hydrothermal waves and stationary rolls in the thermocapillary convection in a rectangular pool. Schwabe et al. ${ }^{14}$ and Ezersky $^{15}$ found that the roll structure in annular pools transforms from single-vortex structure into multi-vortex structure before the hydrothermal wave was formed. The multi-vortex convection also oscillates after the appearance of the hydrothermal wave, but the interaction between the rolls and hydrothermal waves is unclear.

$\mathrm{Xu}$ and Davis ${ }^{16}$ extended the HTW theory to an infinite liquid bridge. In a finite liquid bridge on the ground, azimuthal waves are found by Schwabe et al., ${ }^{17}$ Preisser et al., ${ }^{18}$ and Kang et al. ${ }^{19}$ However, in a long liquid bridge, hydrothermal waves and Bénard cells form in space experiments in the sound rocket ${ }^{20}$ and International Space Station (ISS), ${ }^{21,22}$ i.e., MEIS(2008-2013) and Dynamic Surf (2013-2016). ${ }^{23}$ Recently, another ongoing space experiment involving a liquid bridge is under way in China's manned space shuttle TG-2. In addition, secondary instability which transits from spatially periodic steady flow to oscillatory flow was found in a low-Prandtl-number liquid bridge in numerical simulation ${ }^{24}$ and theoretical analysis.

The annular pool with a cooling central column can generate various patterns. This model is simplified from the Czochralski crystal growth, in which the central seed crystal has a lower temperature. Spoke patterns have been observed in the crucible melt with a small Prandtl number. ${ }^{26,27}$ Azami et al. ${ }^{28}$ found a spoke pattern rotating in a thin layer $(3 \mathrm{~mm})$ and drifting polygonal cells in a thick layer $(200 \mathrm{~mm})$. For convenience of observation, transparent fluids with moderate or large Prandtl numbers were used in experiments. Garnier and Chiffaudel ${ }^{29}$ investigated the pattern-forming instability of center-heated pools by using silicone oil of $\operatorname{Pr}=10$. Concentric rolls and hydrothermal waves of type I were observed when $d=1.9 \mathrm{~mm}$. However, for $d=1.2 \mathrm{~mm}$, hydrothermal waves of type II first appeared and then hydrothermal waves of type I appeared. The hydrothermal wave of type I was a spiral wave pattern, and the hydrothermal wave of type II was a localized bidimensional wave. $\mathrm{Li}$ et al. ${ }^{30}$ considered the thermocapillary convection with $\mathrm{Pr}=10-30$ and liquid depth $d=1.5-8 \mathrm{~mm}$ in their experiment. They have found that hydrothermal waves and concentric multi-rolls occurred in the thin layer and bud-shaped and straight spoke patterns appeared in the deep layer. Yu et al. ${ }^{31}$ studied the thermocapillary convection of binary liquid, in which a hydrothermal wave pattern with a source and a sink was observed. Shen et al. ${ }^{32,33}$ investigated the effect of wall rotation to the thermocapillary convection.

The annular pool with a heating central column has been studied extensively. Schwabe ${ }^{14}$ studied the thermocapillary convection of ethanol $(\operatorname{Pr}=17)$. They found two wave modes, which are short wave when $h<1.4 \mathrm{~mm}$ and long wave when $h>1.4 \mathrm{~mm}$. They asserted that this short wave is a hydrothermal wave. Garnier and Chiffaudel $^{29}$ also studied the annular models with heating central columns with silicone oil $(\operatorname{Pr}=10)$. They found that concentric rolls and spiral waves are more localized near the hot center. In addition, a "flower wave" was found near the center with $d=1.9 \mathrm{~mm}$. Recently, Kang et al. ${ }^{34}$ observed standing waves with $m=0,1$, $5,6,7,8$, and 9 and the spiral rotating waves in a central heating pool $(\mathrm{Pr}=16)$, which provide a terrestrial test for the space experiment in SJ10. They also observed the transition routes, including quasi-periodic bifurcation, period-doubling bifurcation, tangent bifurcation, and a combination route of them, in another terrestrial experiment.

On the ground, the complex interaction between thermocapillary and buoyancy should be considered. Besides, oscillation can hardly be observed in the thick layer $(d>3 \mathrm{~mm})$ due to the stabilizing effect of buoyancy. ${ }^{29}$ Several microgravity experiments have been conducted to study the thermocapillary convection in the open annular pool. Schwabe et al. ${ }^{36,37}$ carried out the space experiment MAGIA (Marangoni Grown Instabilities in an Annulus) on the Russian satellite FOTON-12. They observed unstable traveling waves, standing waves, two opposite wave transitions from the source, and traveling waves within the local area. Kamotani carried out another series of space experiments on annular thermocapillary convection with a heated center. In the first space experiment, ${ }^{38}$ they observed only stable convection. However, in the second space experiment, ${ }^{39,40}$ a two-lobed rotating pattern (traveling waves, $m=2$ ) occurs in critical condition, while two-lobed pulsating patterns (standing waves, $m=2$ ) and three-lobed pulsating or rotating patterns (standing waves, $m=2$ and 3) are observed in supercritical condition. The experiments are conducted in pools with fixed aspect ratios but different diameters $D=12 \mathrm{~mm}, 20 \mathrm{~mm}$, and $30 \mathrm{~mm}$. Since the critical $\mathrm{Ma}_{\mathrm{c}}$ is dependent on the diameter $D$, they conjectured that the onset is influenced by surface deformation.

Numerical simulations and ground experiments have been carried out and compared with the space experiments. Sim et al. ${ }^{41}$ and Li et al. ${ }^{42}$ compared their numerical simulation to Schwabe's experiments. Sim et al. ${ }^{41}$ obtained the azimuthal wave pattern, which was in good agreement with the space experiment. But the quantitative agreement of the onset was only achieved by assuming that the free surface is heated by the surroundings. However, Li et al. ${ }^{42}$ carried out simulations with an adiabatic surface, and they found a satisfactory agreement between the simulation and Schwabe's experiment. To compare with Kamotani's experiment, Sim et al. ${ }^{43,44}$ conducted simulation which validated the wave pattern in both flat surface and concave surface. They also indicated that the variation of $M a_{c}$ in the experiment may be caused by the heat loss from the surface instead of surface deformation. Moreover, Lavalley ${ }^{45}$ carried out numerical simulation and ground experiments with the model that is similar to that of Kamotani. They visualized the azimuthal wave with the wavenumber $m=2$ or 3 and validated the wave pattern in the simulation. However, both Lavalley's experiment and simulation have shown that the azimuthal wave was a standing wave near the onset and transition to the traveling wave in supercritical condition. Their pattern transition process is controversial with Kamotani's experiment. ${ }^{39,40}$ 
Although some patterns have been found in the ground and space experiments, the nonlinear dynamics of pattern transition with a curved surface (meniscus), to the best of our knowledge, have only seldom been studied. Curved meniscuses are the most common surface configurations in microgravity due to the surface tension. Since the curvature depends on the volume ratio of the liquid, its effect on thermocapillary convection is also known as volume ratio effects. However, for simplicity, space experiments mainly concern the thermocapillary convection with a flat surface. Only two space experiments with "shallow" and "deep" concave surfaces are mentioned by Kamotani. Since oscillation was not observed in the deep meniscus, Kamotani conjectured that the concave surface can stabilize the oscillation of thermocapillary convection. This was validated in the three-dimensional simulation by Li et al., ${ }^{42}$ in which the critical Re number increases as the volume ratio decreases. They found a two-lobed pulsating wave with a flat surface and a three azimuthal wave with a concave surface $V r=0.81$. The transition of wave was found in their simulation during the increase in $R e$. The effects of volume ratio on the pattern formation and its transition have not been studied yet.

Pattern transition concerns the nonlinear competition and evolution of wave modes as the boundary condition or control parameter changes. Annular pools provide a physical system suitable for the study of pattern transition because they are convenient to make a full observation of the surface wave from the top view. So far, traveling waves, standing waves, and even spiral waves with different wavenumbers have been found, but their emerging conditions and transition process, which are relevant to the spatio-temporal chaos, have not seen much study. In addition, affected by gravity, most experiments obtained unstable patterns with defects such as wave sources or wave sinks. Even in the microgravity experiments, unstable waves have been commonly observed in MAGIA and only a few groups of Kamotani's experiment have recorded regular patterns. Regular waves and wave transition should be further investigated in addition to the volume ratio effect.

China's scientific satellite SJ10, which carried out more than 20 scientific studies, was launched in $2016 .{ }^{46}$ A thermocapillary experiment was carried out in an open cylindrical annulus on the satellite SJ-10. An onboard payload provided temperature and liquid control for establishing thermocapillary convection in specific volume and comprehensive measurements, including thermocouples, an infrared camera, and a laser displacement sensor for measuring oscillation and pattern transition. ${ }^{34}$ Based on the experimental data collected via the infrared camera, the nonlinear competitions and transition dynamics of wave patterns in the thermocapillary convection will be discussed in this paper. The experimental device and the parameters are introduced in Sec. II. In Sec. III, the surface configurations under different volume ratios calculated by the Laplace-Young equation and the basic flow are observed through the infrared image. The regular oscillatory pattern and its transition are researched by a series of frames and a time-evolution graph in Sec. IV. Plenty of patterns and transitions are identified with the effect of volume ratio.

\section{EXPERIMENT METHOD}

\section{A. Experimental device}

The thermocapillary convection experiments on SJ10 are conducted in an annular pool, which is heated by the center column and cooled by the annulus side wall. The diameter of the central column is $d=8 \mathrm{~mm}$, the diameter of the pool is $D=40 \mathrm{~mm}$, and the depth is $H=12 \mathrm{~mm}$. Wedges are designed on the top of the annulus pool and the central column. The wedges are used to pin the contact line of the liquid by the cusp, which prevents the fluid from climbing out of the pool under microgravity condition. Furthermore, the side of wedges and the walls outside the pool are treated with the antiwetted coating film, 3M EGC-1700. The coating film increases the contact angle between the silicone oil and the copper from nearly $0^{\circ}$ to $47^{\circ}$. A top column with diameter $d_{\text {top }}=5 \mathrm{~mm}$ is designed on the heating column and coaxial with it. Once the inner wedge fails to pin the contact line, the liquid will climb to the top column, which plays the role of a heater.

The central column is made of copper with a high thermal conductivity. It is heated to a higher temperature $T_{\mathrm{h}}$ by a resistance wire heating film. The annulus wall, also made of copper, was uniformly refrigerated to a lower temperature $T_{\mathrm{L}}$ by 6 semiconductor cooling plates. The temperature difference is controlled precisely according to the predetermined program. As the experimental fluid is injected between the central column and the annulus wall, the temperature gradient is established in the fluid, which induces the thermocapillary convection. The experimental fluid is 2 cSt Kf-96 silicone oil made by Shin-tsu, and its physical property is shown in Table I. The silicone oil is stored in a cylinder and injected into the pool by using a stepper motor when conducting the space experiment. The injection hole is located at the bottom of the pool near the central column. The speed of the motor is controlled as low as $0.008 \mathrm{~mm} / \mathrm{s}$ to avoid liquid jet or surface sloshing during the injection. The variation of free surface during the injection is recorded by using a CCD camera (Fig. 1).

After the liquid reaches a specific volume ratio, the device starts to exert temperature difference in the liquid pool according to the

TABLE I. The physical property of KF96 2 cSt silicone.

\begin{tabular}{lccccccc}
\hline \hline & & & $\begin{array}{c}\text { Coefficient } \\
\text { of thermal } \\
\text { Kinematic } \\
\text { viscosity }\end{array}$ & $\begin{array}{c}\text { Thermal } \\
\text { Density }\end{array}$ & $\begin{array}{c}\text { Coefficient } \\
\text { of surface } \\
\text { tension }\end{array}$ & $\begin{array}{c}\text { Temperature } \\
\text { coefficient of } \\
\text { surface tension }\end{array}$ & $\begin{array}{c}\text { Prandtl } \\
\text { number }\end{array}$ \\
Silicone oil & $v\left(\mathrm{~m}^{2} / \mathrm{s}\right)$ & $\rho\left(\mathrm{kg} / \mathrm{m}^{3}\right)$ & $\alpha\left(1 /{ }^{\circ} \mathrm{C}\right)$ & $\kappa\left(\mathrm{m}^{2} / \mathrm{s}\right)$ & $\sigma(\mathrm{N} / \mathrm{m})$ & $\sigma_{\mathrm{T}}\left[\mathrm{N} /\left(\mathrm{m}{ }^{\circ} \mathrm{C}\right)\right]$ & $\operatorname{Pr}$ \\
\hline $2 \mathrm{cSt}$ & $2.00 \times 10^{-6}$ & $8.7 \times 10^{2}$ & $1.24 \times 10^{-3}$ & $7.12 \times 10^{-8}$ & $1.83 \times 10^{-2}$ & $-7.15 \times 10^{-5}$ & 28.1 \\
\hline \hline
\end{tabular}




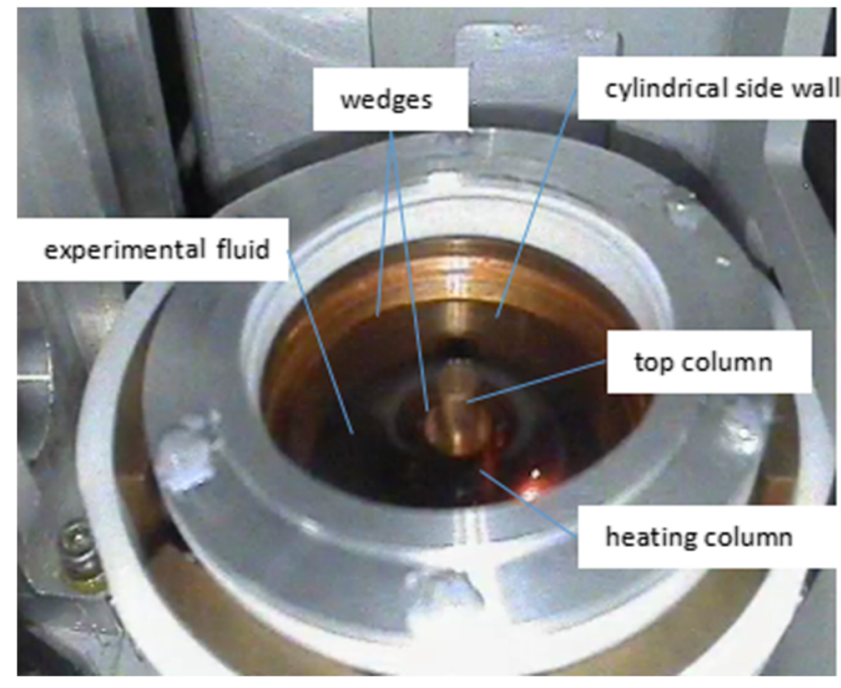

FIG. 1. Annular pool in SJ10.

experimental program. The central column is heated to a target temperature in linear or several steps, and the annulus wall is controlled to $1{ }^{\circ} \mathrm{C}$ lower than the ambient temperature. The oscillation of thermocapillary convection is diagnosed by using thermocouples, a displacement sensor, or an infrared camera. The temperature oscillation of thermocapillary convection is measured by using 6 thermocouples, which are located near the central column at two horizontal planes. The surface oscillation is measured by using a laser displacement sensor with sensitivity to micron meter. A FLIR infrared camera is used (module Tau2, sensitivity: $0.05 \mathrm{C}$, frame rate: $7.5 \mathrm{fr} / \mathrm{s}$ ) to capture the temperature distribution of thermocapillary convection. The combination of various measurements improves the accuracy for judging the critical temperature difference, $\Delta T_{c}$. Infrared images give a very intuitive observation of the hydrothermal wave, which makes it convenient to observe the competition and evolution of wave patterns.

The SJ-10 satellite has been working for 17 days in orbit. The cumulative operating time of our experiments is $74 \mathrm{~h}$, and 23 space experiments are finished. The volume ratio and the heating program are adjustable in space experiments. The volume ratio ranges from 0.363 to 1.220 . The temperature difference is set in the range of $25-40{ }^{\circ} \mathrm{C}$, which is expected higher than the critical temperature difference to observe the oscillation. As a result, we have successfully established thermocapillary convections and observed oscillations in all space experiments.

\section{B. Experimental parameters}

Under the microgravity condition, the main dimensionless numbers of pure thermocapillary flow are shown as follows:

$$
\begin{gathered}
M a=\frac{(\partial \sigma / \partial T) R_{o} \Delta T}{\rho v \kappa}, \\
\operatorname{Pr}=\frac{v}{\kappa}
\end{gathered}
$$

$$
\begin{gathered}
B i=\frac{\alpha H}{\lambda}, \\
A r=(D-d) / 2 H, \\
R r=d / D, \\
V r=V / V_{0},
\end{gathered}
$$

where $\rho$ represents the liquid density, $\kappa$ represents the thermal diffusivity, $v$ represents the kinematic viscosity, $\alpha$ represents the coefficient of thermal expansion, $\partial \sigma / \partial T$ represents the temperature coefficient of surface tension, and $\Delta T$ represents the temperature difference.

The Marangoni number $M a$ is the ratio between thermocapillary force and thermal diffusion, which characterizes the strength of the thermocapillary effect. The maximum of temperature difference corresponds to $M a$ of $4.6 \times 10^{5}$. The Prandtl number $\operatorname{Pr}$ is the ratio of viscous to thermal diffusivity. The $\operatorname{Pr}$ number of $2 \mathrm{cSt}$ silicone oil is 28 . The Biot number $B i$ is used to characterize the surface heat transfer. As buoyancy convection is absent in microgravity, the heat transfer is greatly reduced and the Biot number is very small. $R r$ and $A r$, which represent the radius ratio and aspect ratio of the pool, are 0.2 and 0.75 , respectively. The volume ratio corresponds to different surface configurations, which will be discussed in Sec. III A.

\section{SURFACE CONFIGURATION AND BASIC FLOW}

\section{A. Free surface configuration}

Unlike a horizontal interface in the normal gravity, a meniscus is formed in the microgravity condition due to the capillary effect. In our annular pool, a meniscus is formed between the central column and the outer wall. Contact lines are expected to be pinned by welldesigned wedges on the central column and the annulus pool at the height $H=12 \mathrm{~mm}$. The initial static capillary surface satisfies the axisymmetric Laplace-Young equation, that is,

$$
\sigma\left\{\frac{z^{\prime \prime}}{\left[1+\left(z^{\prime}\right)^{2}\right]^{3 / 2}}+\frac{z^{\prime}}{r\left[1+\left(z^{\prime}\right)^{2}\right]^{1 / 2}}\right\}=\Delta p_{0},
$$

in which $z^{\prime}$ represents $\mathrm{d} z / \mathrm{d} r$ and $z^{\prime \prime}$ represents $\mathrm{d}^{2} z / \mathrm{d} r^{2}$. When the Capillary number $\left(\mathrm{Ca}=\sigma_{\mathrm{T}} \Delta T / \sigma_{0}\right)$ is small, the surface dynamic in thermocapillary convection can be neglected. Most onset and transition of the experiment are with $\Delta T<25$, and the corresponding $\mathrm{Ca}<0.12$. Sim et al. ${ }^{47}$ considered the surface deformation caused by thermocapillary convection, which is $O(-4)$ if $C a=0.1$. Therefore, Eq. (7) is approximately available for the surface configuration under thermocapillary convection. We used a laser displacement sensor to detect surface oscillation in the space experiment. A tiny surface oscillation with the order of micrometers was detected. Such tiny oscillation is negligible when the basic surface configuration is concerned.

The boundary condition of Eq. (7) requires classified discussions.

(1) The first case corresponds to the moderate volume ratio under which both the inner contact line and outer contact line are pinned by the wedges. The boundary condition is 


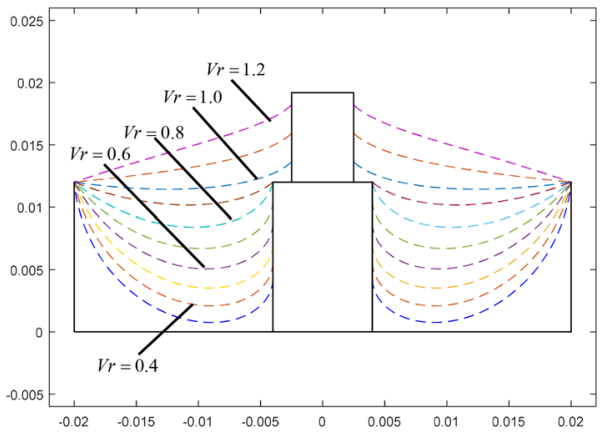

(a)

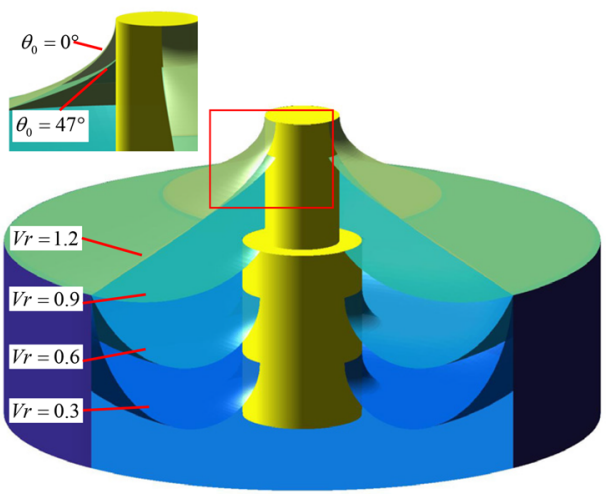

(b)

$$
\left.z\right|_{r=D / 2}=H,\left.\quad z\right|_{r=d / 2}=H,\left.\quad \frac{d r}{d z}\right|_{r=d / 2, z=H}=\tan \theta .
$$

As the volume ratio reduces, both the inner contact angle and the outer contact angle are reduced. The inner contact angle is close to 0 when $V r=0.77$, and the inner contact line moves downward when $V r<0.77$. Therefore, this boundary condition can only be used for $V r>0.77$.

(2) The second case corresponds to $V r<0.77$, under which the inner contact line shrinks downward under the wedge. The boundary condition is

$$
\left.z\right|_{r=\mathrm{D} / 2}=H,\left.\quad z\right|_{r=\mathrm{d} / 2}=\zeta,\left.\quad \frac{\mathrm{d} r}{\mathrm{~d} z}\right|_{r=d / 2, z=\zeta}=0,
$$

where $\zeta$ is the height of the inner contact line.

(3) The third case is that the liquid exceeds over the wedge of the central column and climbs along the top column with a diameter of $5 \mathrm{~mm}$. The boundary condition is

$$
\left.z\right|_{r=\mathrm{D} / 2}=H,\left.\quad z\right|_{r=\mathrm{d} / 2}=\zeta,\left.\quad \frac{\mathrm{d} r}{\mathrm{~d} z}\right|_{r=d / 2, z=\zeta}=\tan \theta_{0} .
$$

Due to the anti-wetting treatment, we consider that the advancing contact angle is $47^{\circ}$ and the receding contact angle is $0^{\circ}$. Therefore, the contact angle $\theta_{0}$ is assigned to $0^{\circ}$ and $47^{\circ}$, respectively.

The Laplace-Young equation can be solved by the shooting method. The numerical simulation of surface configuration is shown in Fig. 2. The 2D diagram of liquid surfaces at volume ratios $V r=0.3$, $0.4, \ldots, 1.2$ is shown in Fig. 2(a), and the $3 \mathrm{D}$ diagram of liquid surfaces at volume ratios $V r=0.3,0.6,0.9$, and 1.2 is shown in Fig. 2(b). It is found that the lowest point of the meniscus is very close to the bottom of the pool at a volume ratio $V r=0.3$. If the liquid continues to be reduced, the meniscus will touch the bottom. In that condition, the liquid will be separated into two isolated volumes, distributed in the inner corner and the outer corner of the pool. This configuration is not in the scope of this experimental study since the radial temperature gradient cannot be established due to the disconnection of liquid.

Table II shows the contact line, contact angles, and capillary pressure of the free-surface configurations at different volume ratios. When $V r>0.77$, the contact lines are pinned by the wedges on the central column and the outer wall. Therefore, as the liquid volume increases, the contact lines are fixed and the contact angles increase. The case of flat surface corresponds to $V r=1$, with both contact angles equal to $90^{\circ}$. If the volume ratio is increased greater than 1 , a "convex" surface will be formed. But, in fact, experiments with $V r>1$ are conducted after the return capsule has been separated from the satellite. The reacting force during the separation causes a very large residual acceleration on the satellite. Unavoidably, the liquid escapes from the pool and some liquid is left on the wedge of the heating column, which results in the wedge losing pinning effect. Therefore, the working fluid submerges the wedge and climbs along the top column for $V r>1$.

Three typical configurations are presented in Figs. 3(a)-3(c), which show the infrared images of the experiments with $V r=0.600$, 0.834 , and 1.053 , respectively. The configurations are distinguished by the inner contact line. Figure 3 (a) shows that the inner contact line does not reach the top wedge because the volume ratio $V r<0.77$. Also, Fig. 3(b) shows that the inner contact is pinned

TABLE II. The contact lines and angles in the annular pool.

\begin{tabular}{lcccc}
\hline $\begin{array}{l}\text { Volume } \\
\text { ratio }\end{array}$ & $\begin{array}{c}\text { Contact angle } \\
\text { (center) }\end{array}$ & $\begin{array}{c}\text { Contact angle } \\
\text { (wall) }\end{array}$ & $\begin{array}{c}\text { Inner contact } \\
\text { line }(\mathrm{mm})\end{array}$ & $\begin{array}{c}\text { Pressure } \\
(\mathrm{Pa})\end{array}$ \\
\hline 0.3 & 0 & 5.8 & 4.29 & -2.37 \\
0.4 & 0 & 12.7 & 5.65 & -2.33 \\
0.5 & 0 & 20.1 & 7.14 & -2.26 \\
0.6 & 0 & 28.9 & 8.786 & -2.13 \\
0.7 & 0 & 40.4 & 10.609 & -1.93 \\
0.77 & 0 & 48.5 & 12 & -1.73 \\
0.8 & 11.8 & 52.1 & 12 & -1.61 \\
0.9 & 50.4 & 69.3 & 12 & -0.96 \\
1 & 0 & 79.8 & 15.838 & -0.59 \\
1 & 47 & 82.1 & 13.628 & -0.44 \\
1.1 & 0 & 93.4 & 18.238 & -0.13 \\
1.1 & 47 & 95.7 & 15.92 & 0.03 \\
1.2 & 0 & 106.5 & 20.659 & 0.31 \\
1.2 & 47 & 108.8 & 18.207 & 0.46 \\
1.25 & 0 & 112.6 & 21.855 & 0.5 \\
1.25 & 47 & 114.9 & 19.331 & 0.65 \\
\hline \hline
\end{tabular}




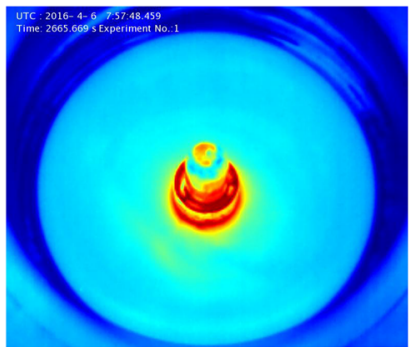

(a) $V r=0.600$

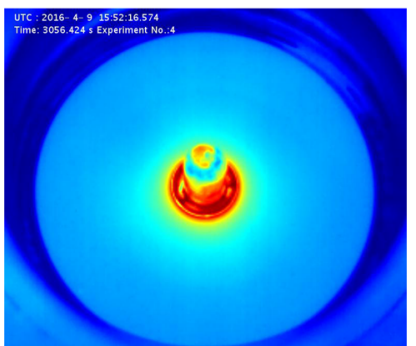

(b) $V r=0.834$

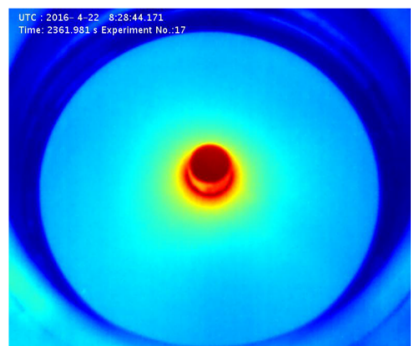

(c) $V r=1.053$
FIG. 3. The infrared images of the liquid surface. by the wedge. Figure 3(c) shows that the inner contact line has exceeded the wedges and attached to the top column. Therefore, these typical configurations are consistent with our numerical prediction.

The inner contact line is characterized by its distance to the bottom, denoted as $\zeta$. The simulations and experiments are shown in Fig. 4, which validate that the surface configurations are classified into three regimes.

(1) Zone $1(V r<0.77)$ : the contact line is lower than the inner wedge $(\zeta<12 \mathrm{~mm})$, and the inner contact angle $\theta i$ is $0^{\circ}$. The contact line $\zeta$ increases with the volume ratio and it reaches the inner wedge $(\zeta=12 \mathrm{~mm})$ when $V r=0.77$.

(2) Zone $2(0.77<V r<1)$ : both the inner contact line and outer contact line are fixed at the wedges, and the meniscus is a concave surface.

(3) Zone $3(V r>1)$ : the wedge fails to pin the fluid and the contact line attaches to the top column. As the advancing contact angle $\left(47^{\circ}\right)$ and the receding contact angle $\left(\approx 0^{\circ}\right)$ are different, simulation of both cases of the contact line is shown in Fig. 4 as two lines. Experimental data lie between these two lines.

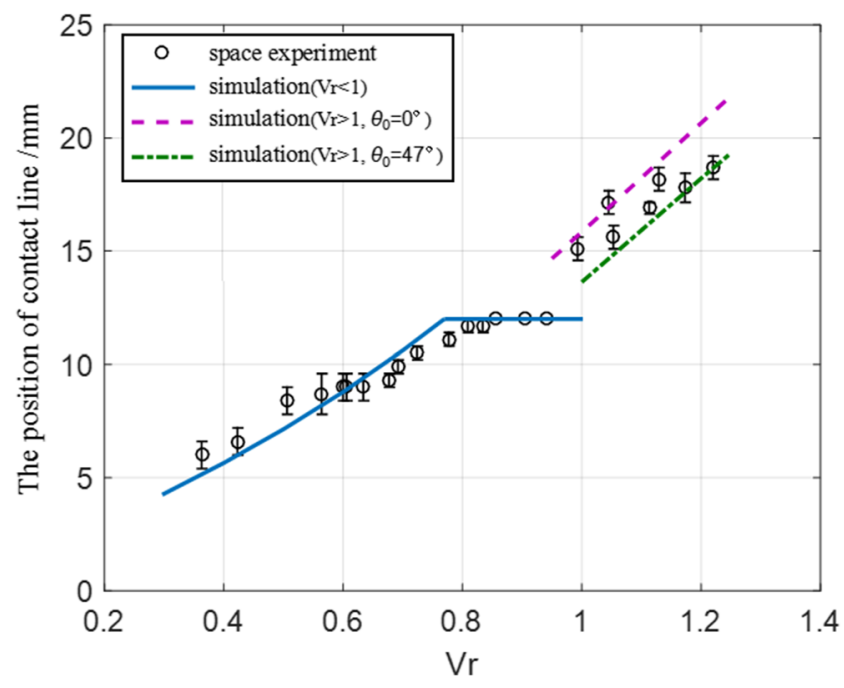

FIG. 4. The volume ratio vs the position of the inner contact line.

\section{B. The basic flow}

Thermocapillary flow couples the temperature field with the velocity field. For a large $\operatorname{Pr}$ number fluid, the flow is determined by the temperature field. Therefore, the feature of thermocapillary convection is characterized by the temperature field. The temperature profile, obtained by using the infrared camera, is an axisymmetric slope, as the center of fluid is heated. The slope is expected as a $T=A \ln (\mathrm{r})+B$ distribution in thermal diffusion without convection. $A$ and $B$ are determined by boundary conditions. Since the surface tension has a negative temperature coefficient, the gradients of the surface tension and the temperature are opposite. As a result, surface tension drives the surface liquid away from the center. Meanwhile, the flow near the bottom moves toward the center as a back flow, which forms a thermocapillary convection. Thermal boundary layers are generated by the strong thermocapillary convection. Figure 5 shows the surface temperature in a $3 \mathrm{D}$ graph. Unlike the profile of thermal diffusion, a steep temperature variation is found near the central column and the annulus side wall.

The temperature profile is affected by the value of the temperature difference which is increasing. However, only the profile configuration is concerned, and a better description is given by a normalized temperature. Here, we define the normalized temperature as $\tilde{T}=\left(T-T_{\text {low }}\right) /\left(T_{\text {high }}-T_{\text {low }}\right)$. Figure 6 shows the normalized temperature profile when $\Delta T=2,4,6, \ldots, 12{ }^{\circ} \mathrm{C}$ and $V r=0.667$. The evolution of temperature profile is clearly observed as the temperature difference increases. When the temperature difference is $2{ }^{\circ} \mathrm{C}$, the temperature profile approximates to thermal diffusion because the convection is very weak. As the temperature difference increases from 2 to $6{ }^{\circ} \mathrm{C}$, the thermocapillary convection is intensified and thermal boundary layers are formed near both the hot wall and the cold wall. The profile in the boundary layers is very steep, while the profile outside the boundary layers is nearly flat. It seems that there exists a saturation when $\Delta T>8{ }^{\circ} \mathrm{C}$ and that the profile converges toward a specific configuration.

From the temperature profile, the vortex structure of basic flow can be assessed. Figure 6(a) gives the profile of single-vortex flow which is the basic flow in most of the space experiments. However, the double-vortex flow has been found in the deep concave meniscus in $V r=0.363$ and $V r=0.420$. Figure 6(b) shows the temperature profile of $V r=0.363$, where two "plateaus" appear. Each "plateau" corresponds to a vortex structure. The boundary between "plateaus" is located at $r=10 \mathrm{~mm}$, which is the deepest position of the 


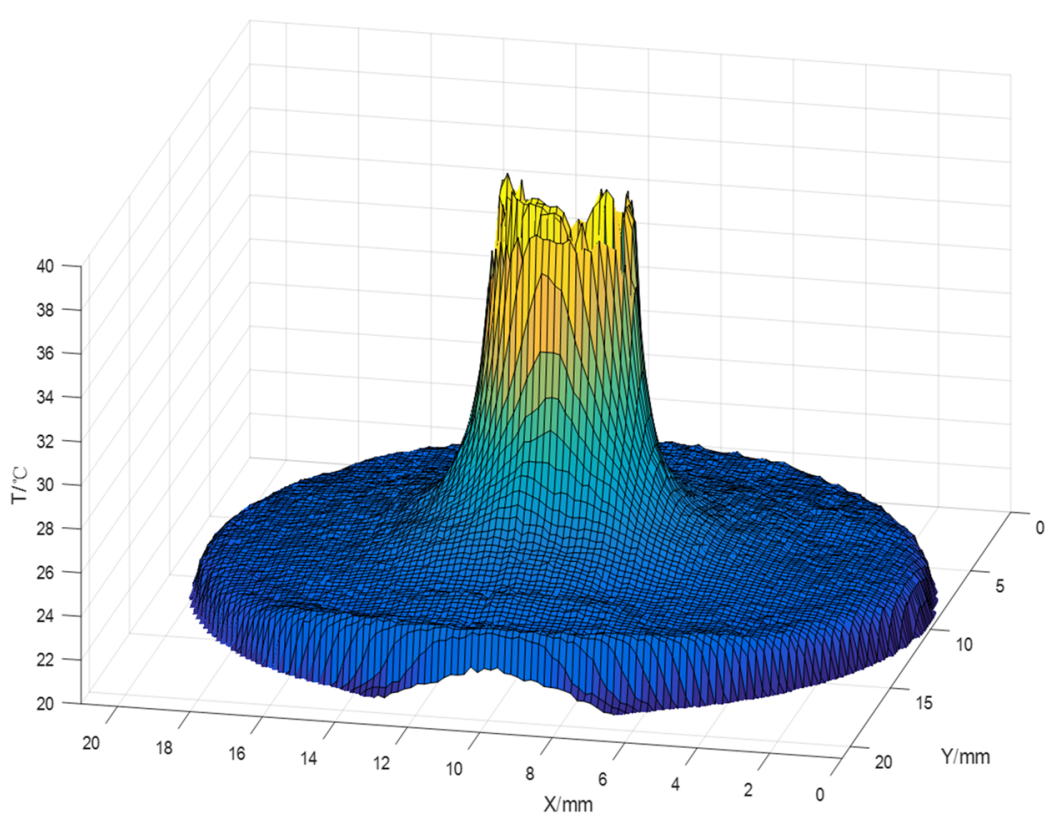

FIG. 5. The temperature distribution on the free surface $(\mathrm{Vr}$ $=0.677, \Delta T=24^{\circ} \mathrm{C}$ ). meniscus. It is conjectured that the single vortex is separated into two vortices by the deep concave surface.

The thickness of the thermal boundary monotonically decreases with the increase in temperature difference. We only focus on the boundary layer near the heating column. Assuming the temperature at the boundary layer distributes in exponential function, $T \sim \exp (-x / \delta)$. $\delta$ is defined as the nominal thickness of the boundary layer, which is obtained by fitting the temperature profile. The nominal boundary layer is not the real boundary layer. For example, although no boundary layer is formed in the thermal diffusion case $(T \sim \ln (\mathrm{r}))$, the nominal thickness of the boundary layer is calculated as $7.15 \mathrm{~mm}$ in our annular pool. So, it is believed that this definition of boundary layer can only be used when $\delta \ll 7.15 \mathrm{~mm}$.

Figure 7 shows the thickness $\delta$ depending on the temperature difference in the experiments. With the increase in the temperature difference, the thickness of the thermal boundary is decreased. Saturation is also found in the thickness of the boundary layer when
$\Delta T>10{ }^{\circ} \mathrm{C}$. The thickness $\delta$ approximates to $1-2 \mathrm{~mm}$ at the large temperature difference. Saturation indicates that the basic flow develops toward a nonlinear configuration. The oscillatory thermocapillary convection occurs at the saturated configuration of basic flow with $\Delta T>12{ }^{\circ} \mathrm{C}$. In addition, the thicknesses of the boundary layer at $V r=0.363$ and 1.129 is greater than that at $V r=0.634$ and 0.94 . This indicates that the minimum of thickness occurs at a moderate volume ratio.

Figure 8 shows the boundary layer thickness depending on the volume ratio. According to the three zones we define in Sec. III A, here, we make a classified discussion about the thermal boundary layers. In zone $1(V r<0.77)$, the thickness of the boundary layer decreases with the volume ratio. In zone $2(0.77<V r<1)$, the thickness of the boundary layer increases with the volume ratio. In zone $3(V r>1)$, the thickness of the boundary layer jumps to a higher value because the liquid exceeds over the wedge. The thinnest boundary layer occurs at the volume ratio $V r \approx 0.8$.

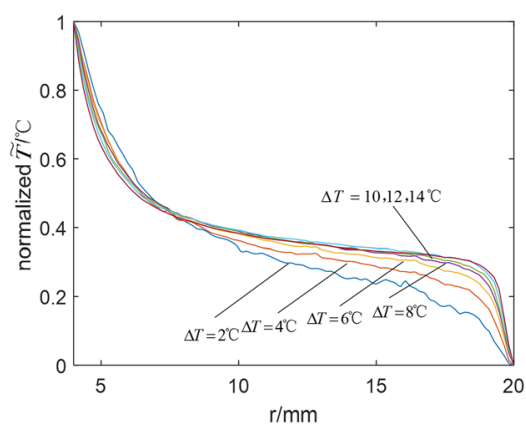

(a) $V r=0.677$

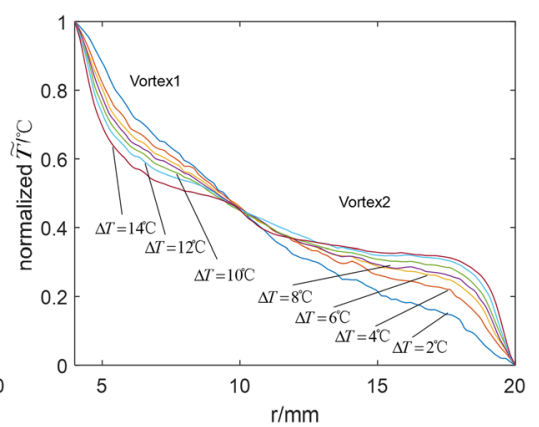

(b) $V r=0.363$
FIG. 6. Normalized temperature distributions at various temperature differences. 


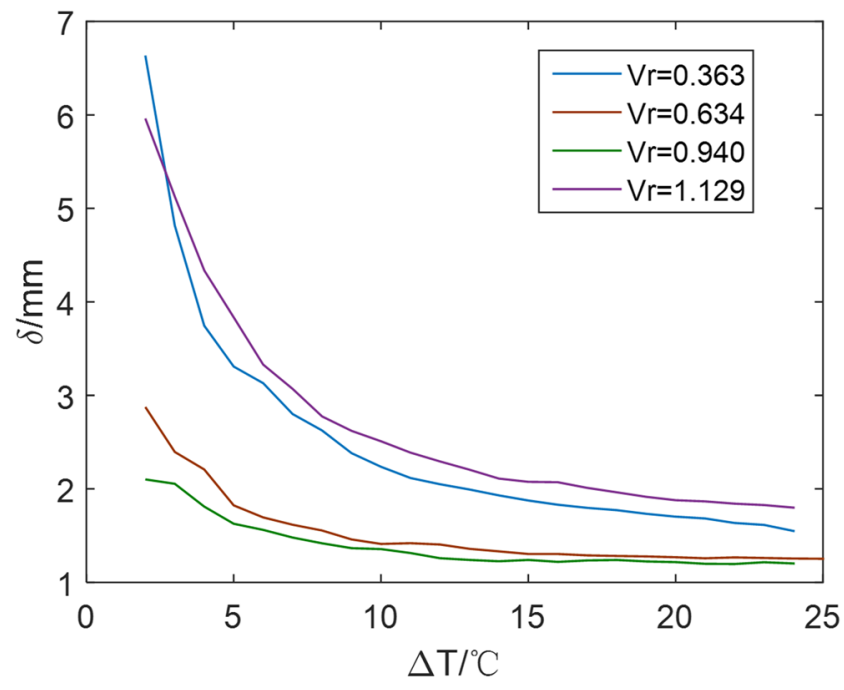

FIG. 7. $\delta$ vs $\Delta T$.

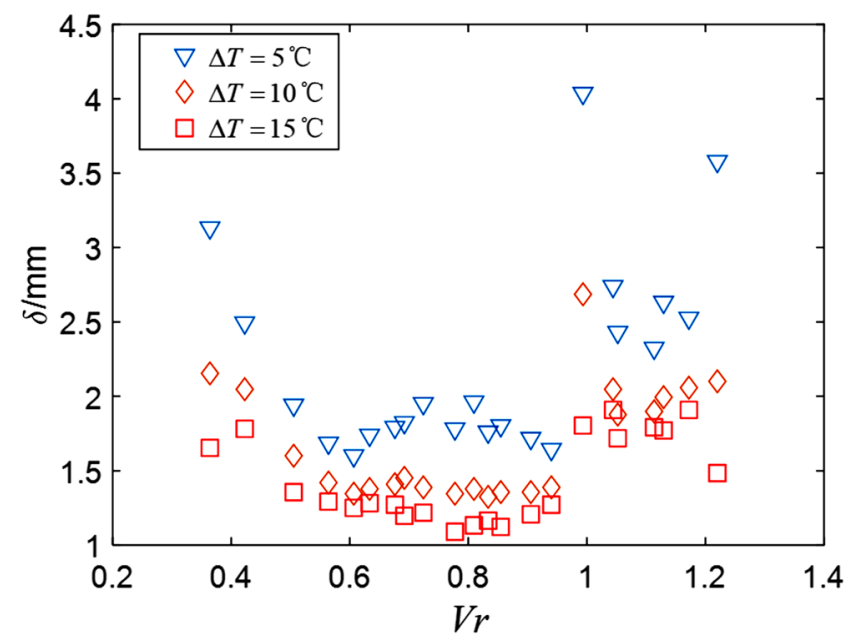

FIG. 8. $\delta$ vs Vr.

\section{THERMOCAPILLARY WAVES}

\section{A. The critical condition of the onset and the transition}

As the temperature profile becomes a nonlinear configuration when $\Delta T>10^{\circ} \mathrm{C}$, the time-independent convection transitions to the oscillatory convection. The oscillatory convection is caused by the appearance of wave when $\Delta T$ reaches a critical condition. The instability of the wave pattern is recorded from the thermal infrared videos. Moreover, the time point of wave appearance is judged by the infrared videos and the critical temperature difference is read from the thermocouples. It has been demonstrated that the critical condition given by this method is in satisfactory agreement with the judgment by the oscillation signal of thermocouples or the surface oscillation.
Once the liquid pool and the fluid properties are fixed, the volume ratio is the critical parameter for the instability of oscillatory thermocapillary convection. In the thermocapillary convection of the liquid bridge, $\mathrm{Hu}^{48}$ has found that the volume ratio effect will influence the onset of oscillation and oscillatory mode. The most obvious change brought by the variation of volume ratio is surface configuration. From the perspective of fluid physics, the instability depends on the basic flow, which is determined by the geometry and boundary conditions. On the one hand, the variation of volume ratio will form a concave or convex surface, which changes the geometry. On the other hand, the driven force, provided by the Marangoni stress from the surface, is sensitive to the surface configuration. Therefore, the volume ratio has a significant influence on the instability of thermocapillary convection.

Our space experiments have studied the volume ratio effect on the critical condition. Figure 9 shows the critical curve depending on the volume ratio effect. As the volume ratio ranges from 0.36 to 1.22 , the critical temperature differences scatter in the range of $\left[12.30{ }^{\circ} \mathrm{C}, 25.86{ }^{\circ} \mathrm{C}\right]$. The critical curve is divided into two branches. The right curve is descending with the increase in volume ratio, and the left curve is ascending with the increase in volume ratio except $V r=0.36$ and 0.42 . As double vortices appear in the experiments with $V r=0.36$ and 0.42 while single vortex occurs in the other experiments, we consider that the decrease of left curve in small volume ratio is probably induced by the change of flow structure.

There is an apparent distinction between the wave patterns of the two branches. The left branch with $V r<0.65$ corresponds to irregular wave patterns. The right branches with $V r>0.65$ correspond to regular wave patterns. A plentiful competition and evolution of wave patterns are found in the regular oscillation corresponding to the right branch. As the wave is confined by boundaries in the radial direction, azimuthal waves are formed with the critical wavenumber $\boldsymbol{m}$ equal to 3 or 4 . The wavenumber selections are correlative with the volume ratio zones, classified by the surface configurations (Fig. 10). The wavenumber

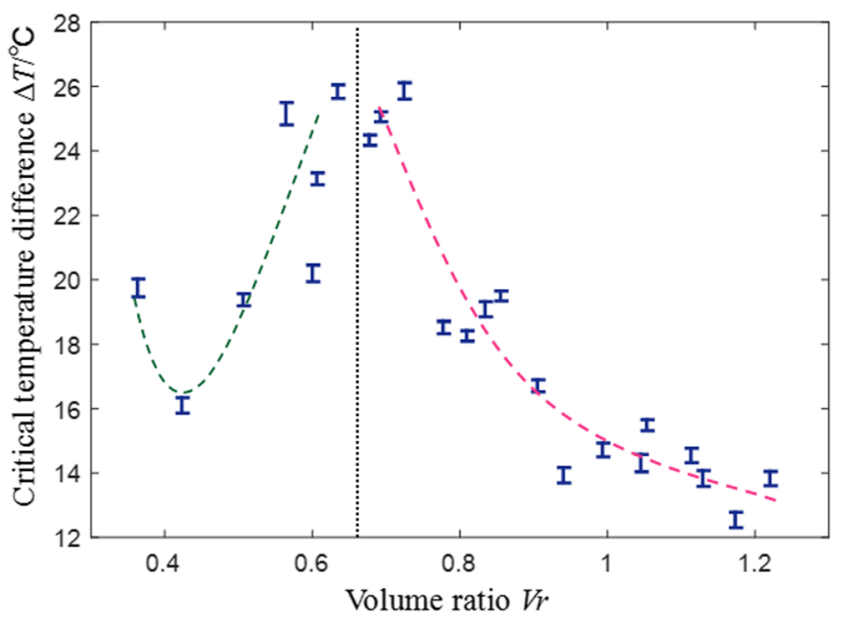

FIG. 9. The critical temperature difference vs $V r$. 


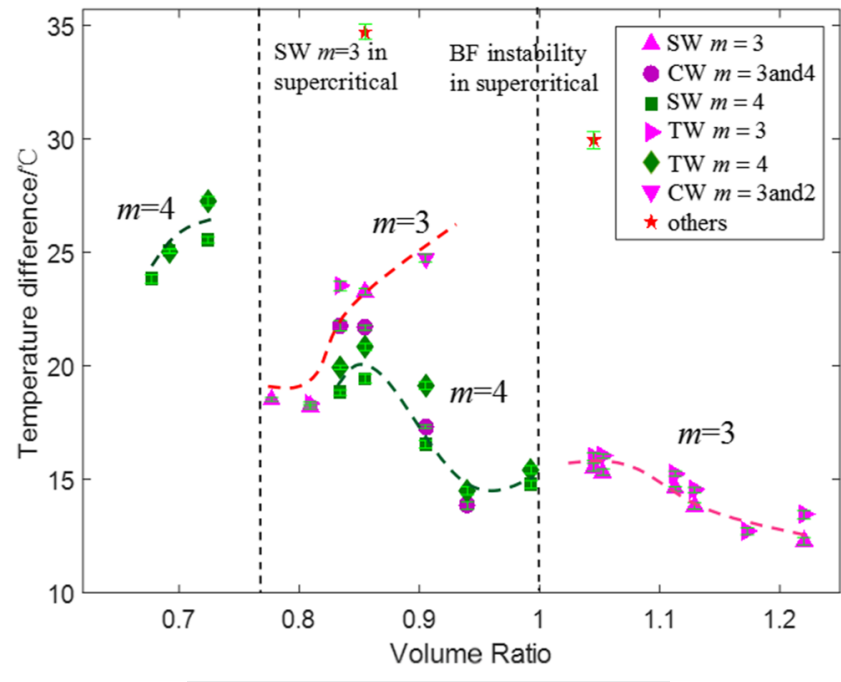

FIG. 10. The competition of $m=3$ and $m=4$.

selections are $m=4$ in zone $1(0.65<V r<0.77)$ and $m=3$ in zone $3(V r>1.00)$. The instability of the wave pattern is dependent on the basic flow. It is inferred that the increase in temperature difference induces the nonlinear change of basic flow. The $m=3$ wave on the basic flow of high temperature is more stable than the $\mathrm{m}=4$ wave.

\section{B. Wave patterns}

The wave patterns are observed by using an infrared camera, which records the surface temperature field. In order to facilitate the observation, image processing is adopted to extract the wave pattern from the basic flow. The temperature field of the basic flow at $t=t 0$ is obtained by time-averaging as

$$
\bar{T}(x, y, t=t 0)=\frac{1}{W} \int_{t=t 0-W / 2}^{t=t 0+W / 2} T(x, y, t) d t,
$$

where $W$ is the time integral window, which is set to 10 times greater than the oscillating period.

The wave is considered as a temperature disturbance superimposed on the basic-flow state. Therefore, the wave disturbance is obtained by subtracting the basic temperature field from the original temperature field

$$
T_{\text {wave }}(x, y, t=t 0)=T(x, y, t 0)-\bar{T}(x, y, t 0) .
$$

The amplitude of wave disturbance is very small, which is in the order of $0.1^{\circ} \mathrm{C}$. We amplify the wave intensity for $\alpha$ times to make an enhancement in visualization

$$
T_{\text {intensify }}(x, y, t=t 0)=\bar{T}(x, y, t 0)+\alpha T_{\text {wave }}(x, y, t 0) .
$$

We have found three types of waves, which are irregular local waves, traveling waves $(m=3$ or $m=4)$, and standing waves/counterpropagating waves $(m=3$ or $m=4)$. Figure 11 shows the irregular local waves at the volume ratio $V r=0.606$ and the temperature difference $\Delta T=25.95{ }^{\circ} \mathrm{C}$. The original graph, the disturbance graph, and the intensified graph are given in Figs. 11(a)-11(c), respectively, and they are displayed by $3 \mathrm{D}$ rendering in Figs. 11(d)-11(f) for better observation. In 3D graphs, it should be noted that the height and the color represent the surface temperature instead of surface deformation. The irregular wave is a non-periodic and non-axisymmetric oscillation in the local area, which occurs in a small volume ratio $(V r<0.65)$. Its critical condition is given by the left branch in Fig. 9, and the oscillation frequency increases with the decrease in volume ratio.

The right branch with $V r>0.65$ in Fig. 9 corresponds to the regular wave pattern with $m=3$ and $m=4$, including traveling

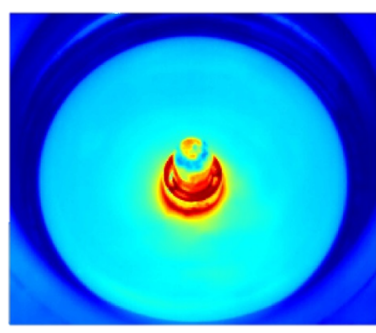

(a)

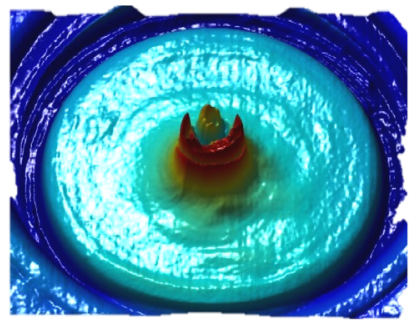

(d)

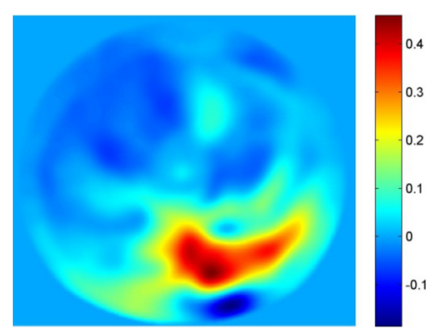

(b)

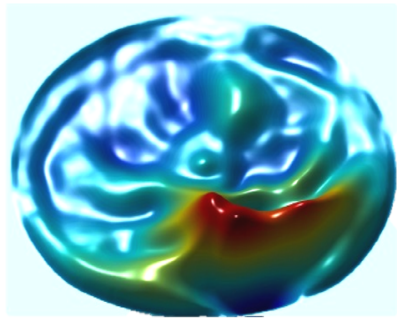

(e)

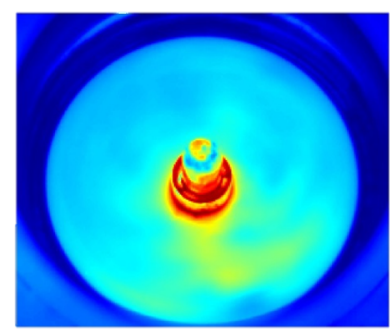

(c)

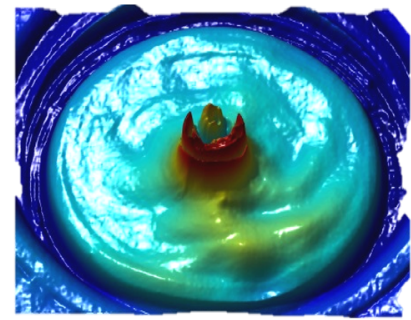

(f)
FIG. 11. An irregular local oscillation pattern. (a) Original IR image (2D). (b) Temperature disturbance (2D). (c) Intensified IR image (2D). (d) Original IR image (3D). (e) Temperature disturbance (3D) (f) Intensified IR image (3D). 


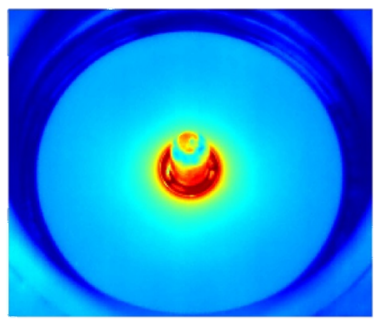

(a)

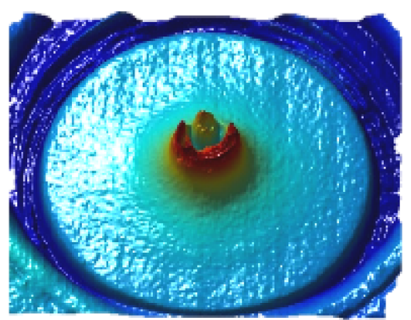

(d)

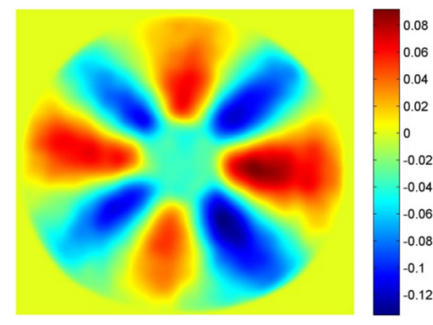

(b)

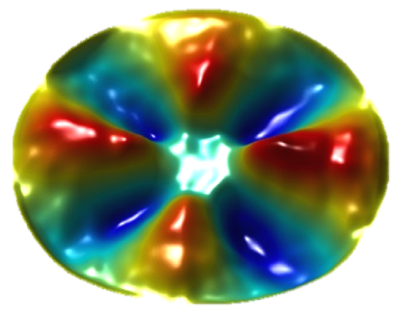

(e)

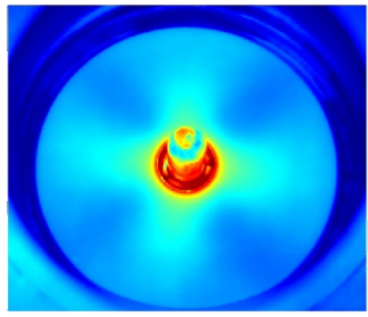

(c)

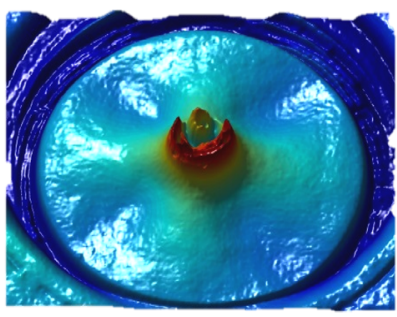

(f)
FIG. 12. The regular azimuthal traveling wave $(m=4)$. (a) Original IR image (2D). (b) Temperature disturbance (2D). (c) Intensified IR image (2D). (d) Original IR image (3D). (e) Temperature disturbance (3D). (f) Intensified IR image (3D). waves, standing waves, and counterpropagating waves. The regular waves are related to a regular sinusoidal oscillation found in the thermocapillary convection. Figure 12 shows the images of a thermocapillary wave with $m=4$ at $\Delta T=17.34{ }^{\circ} \mathrm{C}$ and $V r=0.905$. There is a petal-like axisymmetric pattern with 4 hot petals and 4 cold petals. In the perspective of waves, the wave pattern has four peaks and four troughs [seen in the 3D graph Figs. 12(e) and 12(f)], and its azimuthal wavenumber is $4(m=4)$.

The wave pattern in Fig. 12 is standing waves, which can be observed from the time series of infrared image. The time series at $\Delta T=17.34{ }^{\circ} \mathrm{C}$ is shown in Fig. 13. The period of wave $\mathrm{T}$ is $18.67 \mathrm{~s}$. The initial pattern, as shown in Fig. 13(a), completely repeats at $t=T$, as shown in Fig. 13(e). The wave patterns at $t=0$ [Fig. 13(a)] and $t=T / 2$ [Fig. 13(c)] are pulsating alternately in directions of $45^{\circ}$.
So, the standing waves are also called pulsating waves. When $t=T / 4$ and $t=3 T / 4$, as shown in Figs. 13(b) and 13(d), the amplitude of the surface wave almost decreased to 0 . These features are consistent with the standing wave.

Most of the standing waves appear at the beginning of oscillation (Fig. 13) and quickly transition to the traveling wave. The temperature difference of the transition is shown in Fig. 10, which is close to the onset. For instance, the onset of the standing wave at $V r=0.905$ (shown in Fig. 13) is $\Delta T=17.34{ }^{\circ} \mathrm{C}$ and it transitions to a traveling wave at $\Delta T=19.13{ }^{\circ} \mathrm{C}$. Figure 14 gives the clockwise traveling wave with $m=4$ after the transition at $\Delta T=20.27^{\circ} \mathrm{C}$. Different from the standing wave, the petals of the traveling wave are slightly bent, which is a symmetry break in the azimuth.
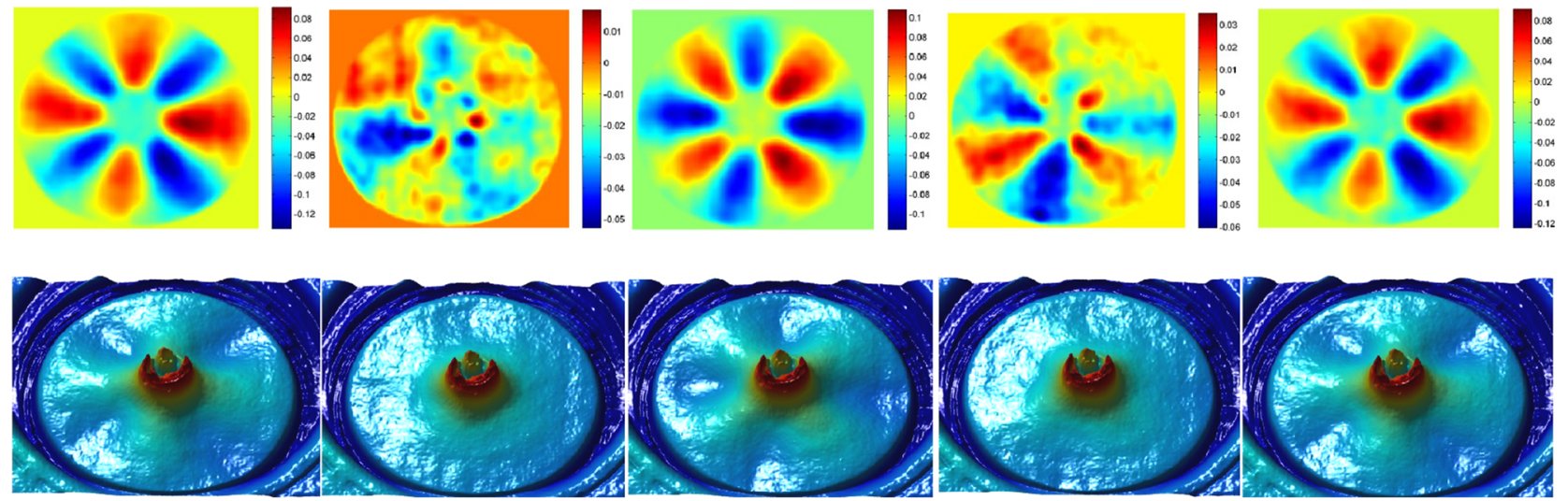

(a) $\mathrm{t}=0$

(b) $\mathrm{t}=\mathrm{T} / 4$

(c) $\mathrm{t}=\mathrm{T} / 2$

(d) $\mathrm{t}=3 \mathrm{~T} / 4$

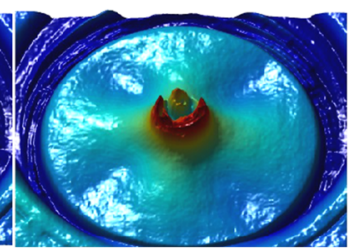

FIG. 13. The standing wave with $m=4$ (period $T=18.67 \mathrm{~s})$. 


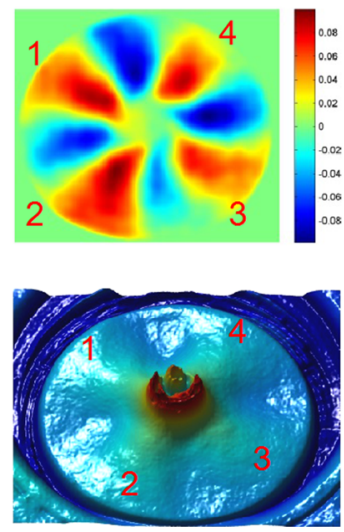

(a) $\mathrm{t}=0$
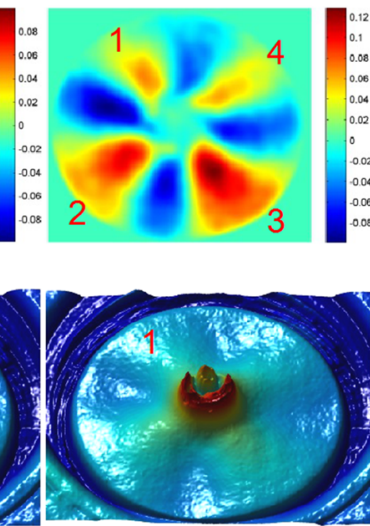

(b) $\mathrm{t}=\mathrm{T} / 4$
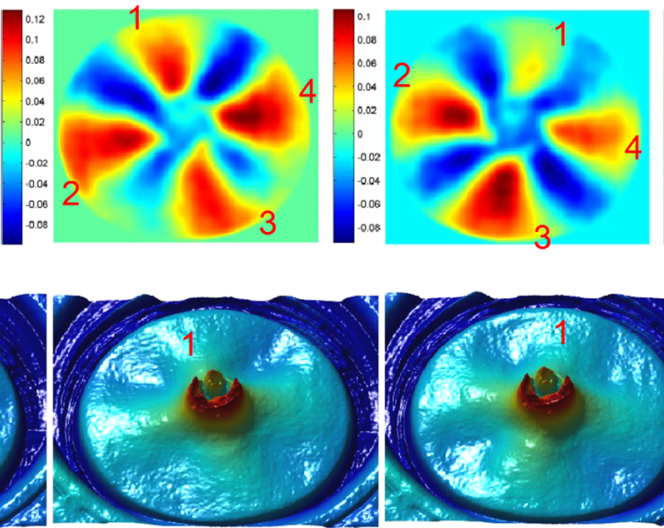

(c) $\mathrm{t}=\mathrm{T} / 2$

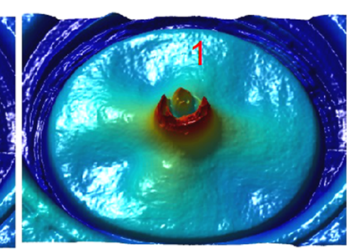

(d) $\mathrm{t}=3 \mathrm{~T} / 4$
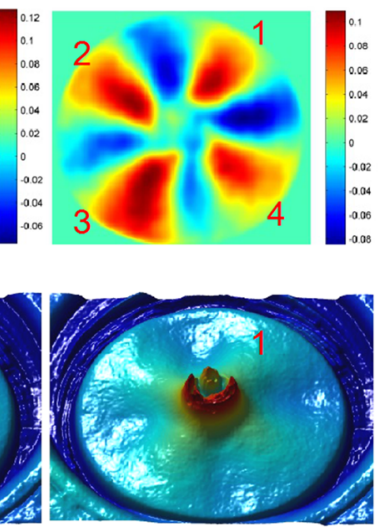

$(\mathrm{e}) \mathrm{t}=\mathrm{T}$

FIG. 14. The clockwise traveling wave with $m=4$ (note: oscillation period $T=20.27 \mathrm{~s}$ ).

For a pure traveling wave, the wave rotates and repeats the initial state in a wave period. The period of the $\mathrm{m}=4$ wave is the time taken by the pattern to rotate $90^{\circ}$. But the amplitudes of the "petals" change during the rotation. For example, the "petals" marked 1 and 4 experience a process as follows: strong $\rightarrow$ weak $\rightarrow$ strong $\rightarrow$ weak $\rightarrow$ strong. This phenomenon is caused by the counterpropagating wave in the anticlockwise direction. Since Fig. 14 is the pattern after the transition from the $m=4$ standing wave to the $m=4$ clockwise traveling wave, the weak anticlockwise wave still remains. Therefore, the pattern presents the feature of a standing wave.

The $m=3$ wave also commonly occurs in the space experiments. In zone 3 , the $m=3$ wave transitions from the standing wave to the traveling wave. In zone 2 , the $m=4$ waves transition to the $m=3$ waves at relatively high temperature differences.
Figure 15 shows the images of the $\boldsymbol{m}=3$ wave at $\Delta T=18.11^{\circ} \mathrm{C}$ and $V r=1.053$. Three peaks and three troughs are formed in the wave pattern. It rotates in an anticlockwise direction, and the petals are also slightly bent similar to the $m=4$ wave in Fig. 14 . Therefore, it infers that the petals are bent toward the opposite direction of the wave.

As two branches are found in the marginal curve as shown in Fig. 9, this phenomenon indicates the competition between different oscillatory modes. The left-branch is the onset of the local irregular wave, and the right-branch is the onset of the azimuthal wave $(\mathrm{m}=3$ or 4$)$. For the azimuthal wave, a slight concave surface leads to a smaller average liquid depth, which increases the viscous force from the bottom. Furthermore, the concave surface has a larger length which may decrease the surface gradient if the temperature is fixed. So, the onset of the azimuthal wave increases with

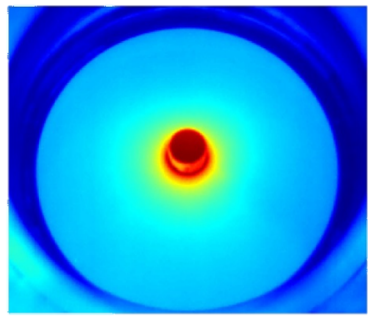

(a)

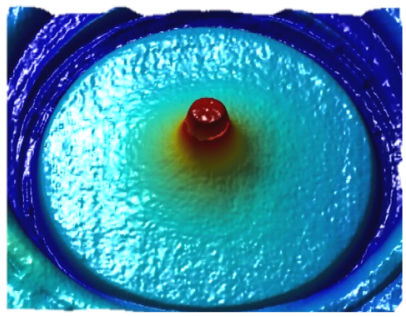

(d)

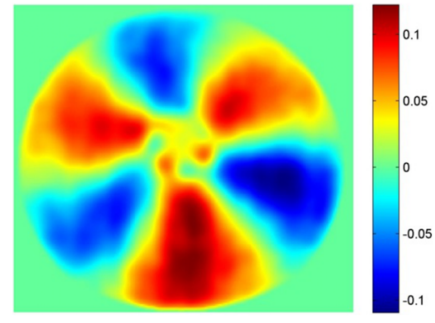

(b)

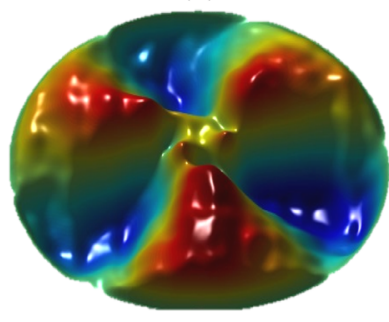

(e)

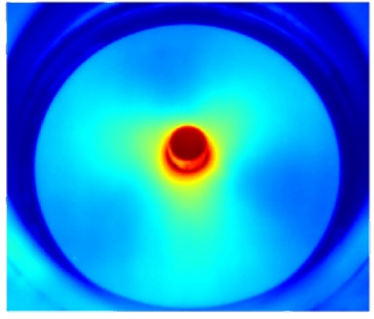

(c)

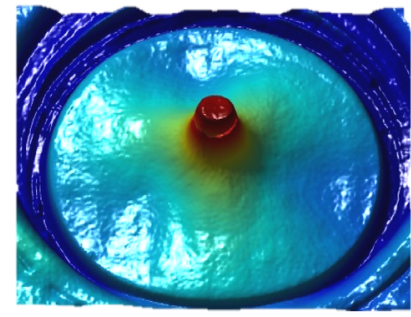

(f)
FIG. 15. The regular azimuthal traveling wave $(m=3)$. (a) Original IR image (2D). (b) Temperature disturbance (2D). (c) Intensified IR image (2D). (d) Original IR image (3D). (e) Temperature disturbance (3D). (f) Intensified IR image (3D). 
the decrease in volume ratio $V r$. However, when the volume ratio decreased to some extent, it forms a deep concave surface with the non-uniformity of depth or temperature gradient. The localized wave is caused by the non-uniformity of surface and presents a lower onset in a deeper concave surface. Therefore, the different tendencies can be explained by the different wave modes depending on the volume ratio.

\section{Pattern transition}

As the temperature difference slowly increases, the critical wave pattern and its transitions occur in the thermocapillary convection. The critical conditions and the corresponding patterns have been shown in Fig. 10. Standing waves are unstable patterns and quickly transition to traveling waves. Traveling waves are stable oscillation patterns, and their critical conditions are slightly higher than that of standing waves. Although stable, the instability of traveling waves occurs in highly supercritical condition. The competition of wavenumbers $m=3$ and $m=4$ in zone $2(0.77<V r<1)$ leads to the transition from the $m=4$ wave to the $m=3$ wave. The traveling wave of $\mathrm{m}=3$ transits to a very stable standing wave in the experiment at $V r=0.905$ with the temperature difference at $34.72{ }^{\circ} \mathrm{C}$. The Benjamin-Feir instability is found in the $m=3$ traveling waves in the experiment at $V r=1.045$ with the temperature difference at $29.94{ }^{\circ} \mathrm{C}$.

\section{Standing wave to traveling wave}

In most of the experiments, the critical wave patterns are standing waves and they transform into traveling waves under weakly supercritical condition. The critical conditions of the standing wave and the traveling wave have been given in Fig. 10. For a better understanding of pattern transition, we introduce a mathematical model to describe the azimuthal wave, which is written as a superposition of the clockwise wave and anticlockwise wave

$T(\theta, r)=f(r)\left[A_{\mathrm{c}} \cos \left(m_{\mathrm{c}} \theta+2 \pi f_{\mathrm{c}} t+\varphi_{\mathrm{c}}\right)+A_{\mathrm{a}} \cos \left(m_{\mathrm{a}} \theta-2 \pi f_{\mathrm{a}} t+\varphi_{\mathrm{a}}\right)\right]$,

where $T(\theta, r)$ describes the temperature distribution on the free surface. The subscripts " $c$ " and "a" represent clockwise and anticlockwise, respectively. $f(r)$ describes the wave profile along the radial direction and satisfies the condition $f(d / 2)=f(D / 2)=0$, the wavenumber $m=3$ or 4 in our experiments, $f$ is the wave frequency, and $\varphi$ is the initial phase.

Traveling waves, standing waves, and counterpropagating waves correspond to a different combination of clockwise waves and anticlockwise waves.

(1) Traveling waves: $A_{\mathrm{c}}>0, A_{\mathrm{a}}=0, m_{\mathrm{c}} \neq 0, f_{\mathrm{c}} \neq 0$ or $A_{\mathrm{a}}>0$, $A_{\mathrm{c}}=0, m_{\mathrm{a}} \neq 0$, and $f_{\mathrm{a}} \neq 0$. This pattern is a single wave traveling in the clockwise or anticlockwise direction.

(2) Standing waves: $A_{\mathrm{c}} \approx A_{\mathrm{a}}, f_{\mathrm{c}} \approx f_{\mathrm{a}}$, and $m_{\mathrm{c}}=m_{\mathrm{a}}$. This pattern is a pair of counterpropagating waves whose amplitudes and wavenumbers are equal. Strictly speaking, standing waves require $A_{\mathrm{c}}=A_{\mathrm{a}}$ and $f_{\mathrm{c}}=f_{\mathrm{a}}$. Here, we loosen the requirements to $A_{\mathrm{c}} \approx A_{\mathrm{a}}$ and $f_{\mathrm{c}} \approx f_{\mathrm{a}}$.

(3) Counterpropagating waves: $A_{\mathrm{c}}>0, A_{\mathrm{a}}>0$, and $m_{\mathrm{c}} \neq m_{\mathrm{a}}$. This pattern is a pair of counterpropagating waves whose wavenumbers are unequal. The counterpropagating waves with $\mathrm{m}=3$ and $\mathrm{m}=4$ are preferred in zone 2 .

The transition from the standing wave to the traveling wave can be described by the evolution of amplitude in Eq. (14). At the critical condition, both the clockwise wave and anticlockwise wave grow exponentially because they are equivalent and unstable. As a result, standing waves $\left(A_{\mathrm{c}} \approx A_{\mathrm{a}}\right.$ and $\left.\mathrm{m}_{\mathrm{c}}=\mathrm{m}_{\mathrm{a}}\right)$ and sometimes counterpropagating waves $\left(A_{\mathrm{c}} \approx A_{\mathrm{a}}\right.$ and $\left.m_{\mathrm{c}} \neq m_{\mathrm{a}}\right)$ appear in the initial oscillation. However, as the amplitudes of the counterpropagating waves increase, nonlinear competition leads to amplification in one wave and attenuation in the other wave. Thus, if one of the wave amplitudes decreases to 0 , the standing waves $\left(A_{\mathrm{c}} \approx A_{\mathrm{a}}\right.$ and $\left.m_{\mathrm{c}}=m_{\mathrm{a}}\right)$ or the counterpropagating waves $\left(A_{\mathrm{c}} \approx A_{\mathrm{a}}\right.$ and $\left.m_{\mathrm{c}} \neq m_{\mathrm{a}}\right)$ develop into a clockwise traveling wave $\left(A_{\mathrm{c}}>0\right.$, $\left.A_{\mathrm{a}}=0\right)$ or an anticlockwise traveling wave $\left(A_{\mathrm{a}}>0, A_{\mathrm{c}}=0\right)$. The direction of wave propagation is in random. We have found that 8 experiments occur clockwise waves and 7 experiments occur anticlockwise waves.

The spatial-temporal evolution diagram of circumferential temperature gives an explicit description of the wave competition. The spatial-temporal evolution of the critical wave in the experiment with $V r=0.855$ is shown in Fig. 16, which presents the transition

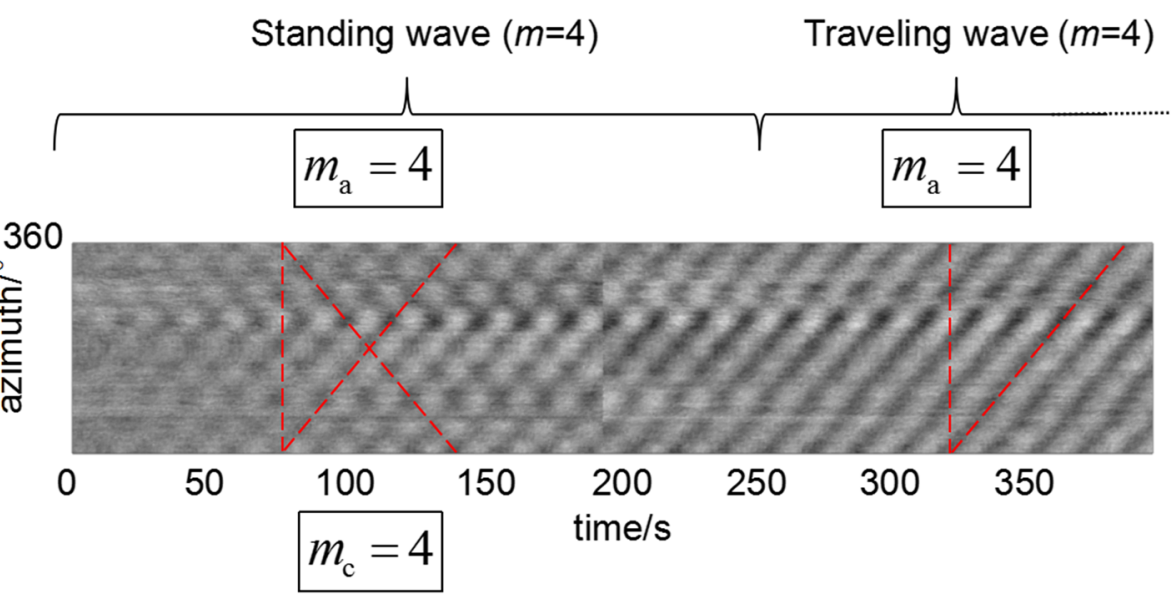



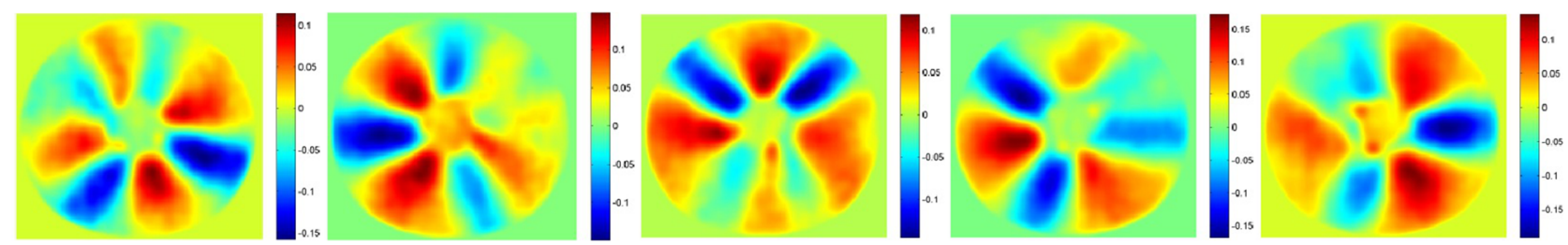

FIG. 17. The transition from the mode with $m=4$ to the mode with $m=3$.

from the $m=4$ standing wave to $m=4$ traveling wave. The $\mathrm{Y}$ axis is the circumferential temperature distribution at $r=10 \mathrm{~mm}$, and the $\mathrm{X}$ axis is the time. The strips oblique downward stand for clockwise waves, and the strips oblique upward stand for anticlockwise waves. Crossed strips indicate two counterpropagating waves, here standing waves. By drawing an oblique line along a strip and a vertical line, the wavenumber is given by counting the number of waves between these two lines. Therefore, the standing wave with $m=4$ is found at the initial oscillation, corresponding to the pattern given by Fig. 13 . Later, when $t>200 \mathrm{~s}$, the clockwise wave decays. When $t>260 \mathrm{~s}$, the anticlockwise wave with $m=4$ dominates the wave pattern, as shown in Fig. 14. Although the anticlockwise wave is dominated, the weak clockwise wave is still found in the spatial-temporal graph. This explains why the petal-like pattern changes its amplitude during the rotation.

There are only two exceptions that the critical pattern is not a standing wave, as shown in Fig. 10. The first exception is in the experiment with $V r=0.94$, in which the counterpropagating waves with $m_{\mathrm{c}}=4$ and $m_{\mathrm{a}}=3$ have the critical wave pattern. The counterpropagating wave is generated by the competition of $m=3$ and $m=4$. In the perspective of waves, the counterpropagating wave and standing wave are equivalent. The second exception happens in the experiment with $V r=1.173$, in which only a clockwise traveling wave is observed as the critical wave. It may be because the standing wave is weak and with a short duration.

\section{The mode with $m=4$ to the mode with $m=3$}

In zone $2(0.77<V r<1)$, the wave pattern is characterized by the competition between the wavenumbers of $m=3$ and 4 .
As a result, plenty of transitions and bifurcations are found in this zone. The critical condition of the $m=3$ wave increases and the critical condition of the $m=4$ wave decreases with the increase in volume ratio in Fig. 10. The gap between the critical conditions of the $m=3$ wave and the $m=4$ wave increases with the increase in volume ratio (shown in Fig. 10).

Figure 17 shows the morphological change during the transition from the $m=4$ wave to the $m=3$ wave in the experiment with $V r=0.834$. During the transition process, one of the 4 petals decays gradually and only 3 petals remain at last. The angle between the peaks is also gradually adjusted from $90^{\circ}$ to $120^{\circ}$. Besides, we found that the wave reverses its direction after the transition from $m=4$ to $m=3$. Similar transitions have been found in $V r=0.855$ and 0.905 experiments, and also the wave changes its direction after the transition.

Figure 18 shows the spatial-temporal evolution of the experiment at $V r=0.834$, in which the wave pattern transitions from $m=4$ to $m=3$. As the temperature difference increases linearly, a weak standing wave pattern with $m=4$ appears in the initial oscillation, which exhibits crossed strips in Fig. 18. Then, the amplitude of the anticlockwise wave is depressed due to the nonlinear effect, and the pattern is predominated by the traveling wave $\left(A_{a} \ll A_{c}\right)$ with $m=4$. Subsequently, the weak anticlockwise wave forms an $m=3$ wave, and the pattern develops into counterpropagating waves with $m_{\mathrm{a}}=3$ and $m_{\mathrm{c}}=4$ when $t>270 \mathrm{~s}$. When $t>550 \mathrm{~s}$, the anticlockwise wave $\left(m_{\mathrm{a}}=3\right)$ intensifies and the clockwise wave $\left(\mathrm{m}_{\mathrm{c}}=4\right)$ decays. Finally, a stable anticlockwise traveling wave with $m_{\mathrm{a}}=3$ is formed after $\mathrm{t}>600 \mathrm{~s}$. Therefore, the transition sequence is as follows: the standing wave $\left(A_{\mathrm{c}} \approx A_{\mathrm{a}}, \mathrm{m}_{\mathrm{c}}=\mathrm{m}_{\mathrm{a}}=4\right)->$ the dominant traveling wave $\left(A_{\mathrm{a}} \ll A_{\mathrm{c}} \cdot m_{\mathrm{c}}=m_{\mathrm{a}}=4\right)->$ the counterpropagating waves

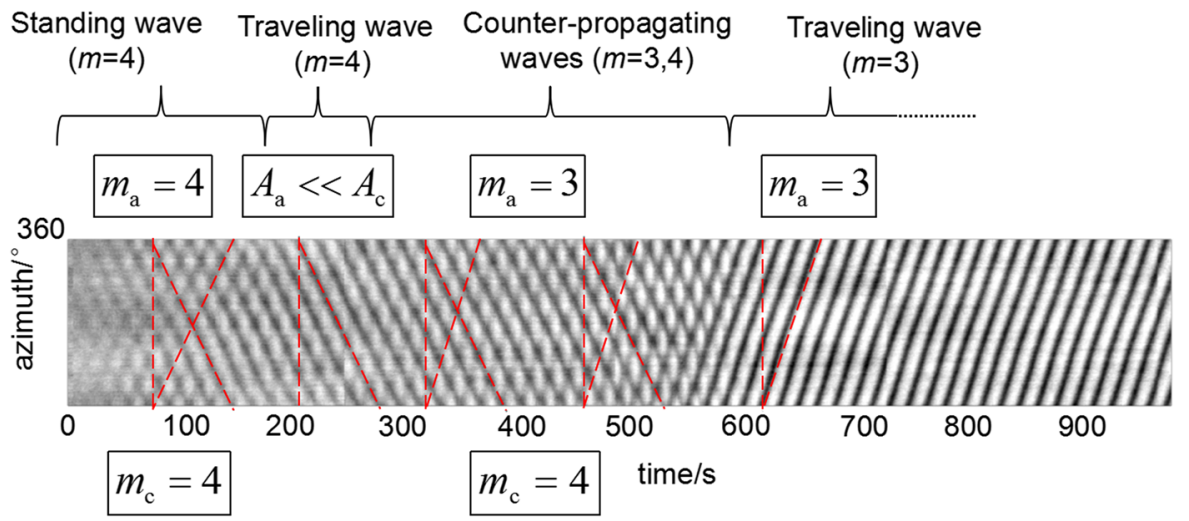

FIG. 18. The evolution from the wave of $m=4$ to the wave of $m=3$. 


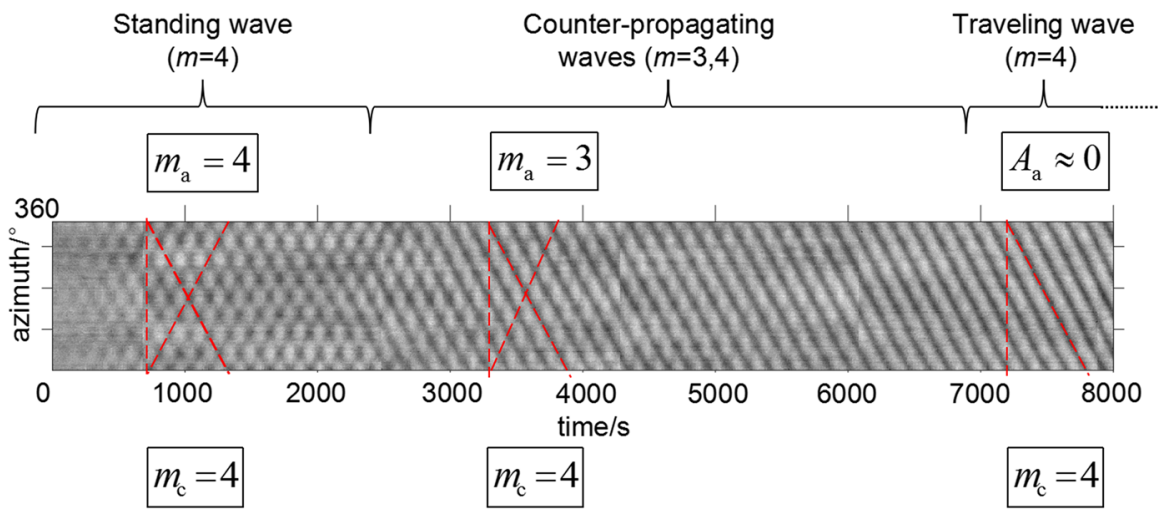

FIG. 19. The competition between the wave with $m=4$ and the wave with $m=3$.

$\left(A_{\mathrm{c}} \approx A_{\mathrm{a}}, m_{\mathrm{a}}=3, m_{\mathrm{c}}=4\right) \rightarrow$ the traveling wave $\left(A_{\mathrm{a}}>0\right.$, $\left.m_{\mathrm{a}}=3\right)$. Also, the corresponding critical conditions are $18.82{ }^{\circ} \mathrm{C}$, $19.93{ }^{\circ} \mathrm{C}, 21.77{ }^{\circ} \mathrm{C}$, and $23.52{ }^{\circ} \mathrm{C}$, respectively. The counterpropagating waves act as a transitional stage for the transition from the $m=4$ traveling wave to the $m=3$ traveling wave. As a consequence, the wave reverses its direction after the transition.

An intuitive assumption is that the wave keeps a constant speed, and the frequency is reduced by $3 / 4$ as the wavenumber decreases from $m=4$ to $m=3$. However, instead of a decrease in frequency, a small increase in frequency from $0.056 \mathrm{~Hz}$ to $0.061 \mathrm{~Hz}$ is found. When the wavenumber decreases, the frequency only changes slightly and the wave speed increases approximately proportional to $1 / \mathrm{m}$. Besides, long period modulation of oscillation amplitude is found in the standing wave and counterpropagating wave near the critical. We infer that the frequencies of the clockwise and anticlockwise waves are different, which cause a beat phenomenon. After developing into the traveling wave, the long-period modulation disappears.

Figure 19 shows the spatial-temporal evolution of the experiment at $V r=0.905$. The competition between $\mathrm{m}=3$ and $\mathrm{m}=4$ finally evolves to the traveling wave with $m=4$. As the temperature difference increases, the transition sequence near the critical is as follows: the standing wave with $m=4->$ the counterpropagating waves $\left(m_{\mathrm{a}}=3, \mathrm{~m}_{\mathrm{c}}=4\right)->$ the clockwise traveling wave with $m_{\mathrm{c}}=4$. In the beginning, $A_{\mathrm{c}}$ and $A_{\mathrm{a}}$ are equally growing, which forms a standing wave. Then, the anticlockwise traveling wave suddenly decreases its wavenumber from $m_{\mathrm{a}}=4$ to $m_{\mathrm{a}}=3$ and forms counterpropagating waves with $m_{\mathrm{c}}=4$ and $m_{\mathrm{a}}=3$. Subsequently, the anticlockwise traveling wave with $m=3$ gradually decays and finally forms a clockwise traveling wave with $m_{\mathrm{c}}=4$.

The stable $m=4$ traveling wave will transform into the $m=3$ traveling wave when $\Delta T=24.75^{\circ} \mathrm{C}$. As shown in Fig. 10, in zone 2 , the critical condition of $m=3$ increases with the increase in volume ratio, but the critical condition of $m=4$ decreases. Therefore, compared with the experiment with $V r=0.834$, a longer duration of $m=4$ traveling wave is found in the experiment with $V r=0.905$. According to the critical curve in Fig. 10, we conjecture that the critical condition of the $m=3$ wave is higher than $25{ }^{\circ} \mathrm{C}$ when $V r>0.905$. Therefore, the transition from $m=4$ to $m=3$ is not found in the experiment with $V r=0.94$. But in its critical wave pattern, counterpropagating waves with $m=3$ and 4 appear, which reveals the existence of the competition between $m=3$ and $m=4$.

\section{The traveling wave to the standing wave}

The transition from the standing wave to the traveling wave is usually found in the initial oscillation, as shown in Fig. 16. The traveling wave is a stable wave pattern, but its transition to the standing wave is found under a relatively high temperature difference in the experiment with $V r=0.855$. The standing wave in supercritical transition is very stable, and the wave patterns are shown in Fig. 20. The temperature difference of the standing wave is $34.72{ }^{\circ} \mathrm{C}$, which is 1.78 times higher than the critical condition. The transition process is presented in the spatial-temporal evolution diagram (Fig. 21). The early oscillation is the stable anticlockwise traveling wave. When $t>200 \mathrm{~s}$, the amplitude of the clockwise traveling wave

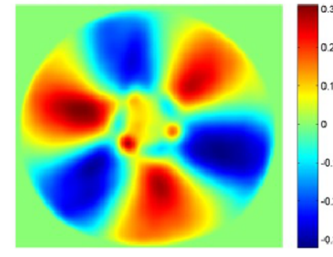

$\mathrm{t}=0 \mathrm{~s}$

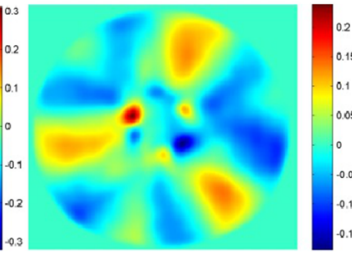

$\mathrm{t}=3.3 \mathrm{~s}$

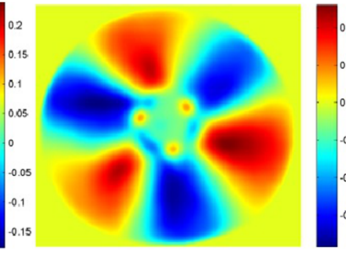

$\mathrm{t}=6.7 \mathrm{~s}$

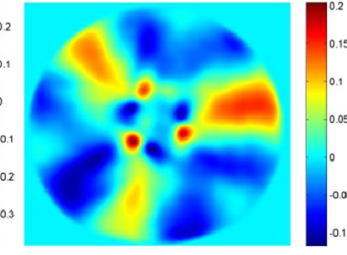

$\mathrm{t}=10.0 \mathrm{~s}$

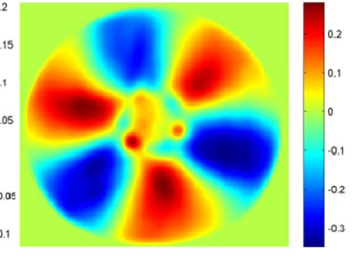

$\mathrm{t}=13.3 \mathrm{~s}$

FIG. 20. The standing wave in supercritical oscillation. 
Traveling wave $(m=3)$

Standing wave $(m=3)$

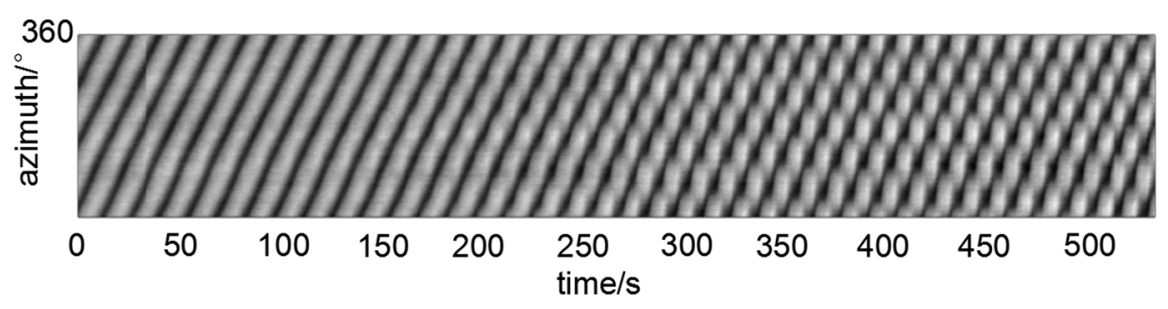

FIG. 21. The spatial-temporal evolution of the transition from the traveling wave to the standing wave.

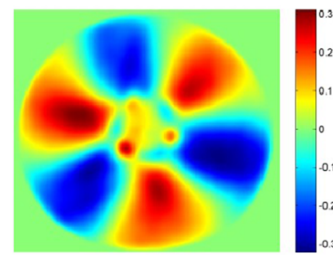

$\mathrm{t}=0 \mathrm{~s}$

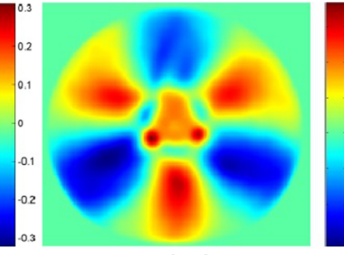

$\mathrm{t}=70.9 \mathrm{~s}$

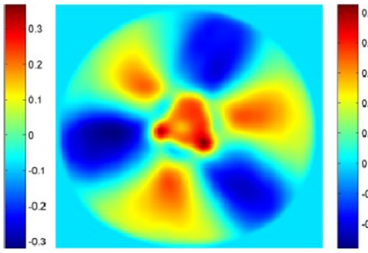

$\mathrm{t}=141.8 \mathrm{~s}$

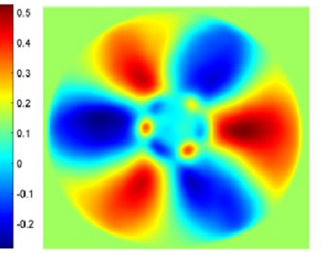

$\mathrm{t}=212.7 \mathrm{~s}$

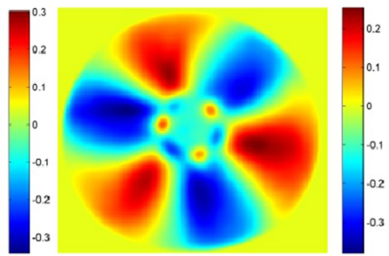

$\mathrm{t}=283.6 \mathrm{~s}$

FIG. 22. The slow rotation of the standing wave.

gradually increases from zero, and the pattern transforms from the anticlockwise traveling wave to the standing wave.

Strictly speaking, the standing wave requires $A_{\mathrm{c}}=A_{\mathrm{a}}$, $m_{\mathrm{c}}=m_{\mathrm{a}}$, and $f_{\mathrm{c}}=f_{\mathrm{a}}$ in Eq. (14). The equality in wavenumbers is easily guaranteed because the wavenumber should be an integral number, $m_{\mathrm{c}}=m_{\mathrm{a}}=3$ in Fig. 22. Nevertheless, frequencies of clockwise and anticlockwise waves are slightly different, with $\Delta f=f_{c}-f_{a} \neq 0$. Without losing generality, the phases $\varphi_{\mathrm{a}}$ and $\varphi_{\mathrm{c}}$ are assumed to be zero. Assuming $A_{\mathrm{c}}=A_{\mathrm{a}}=A$, the mathematical model of counterpropagating waves can be written in the form

$$
\mathrm{F}(\theta, r)=A f(r)\left[2 \cos \left(m \theta+\frac{2 \pi \Delta f}{2} t\right) \cos \left(\frac{2 \pi f_{c}+2 \pi f_{a}}{2} t\right)\right] .
$$

When $\Delta f=0$, Eq. (15) gives a strict standing wave, in which the wave nodes are stationary. However, when $\Delta f \sim o(1)$, Eq. (15) gives a slow modulated standing wave, in which the wave nodes are rotating with an angular velocity, $2 \pi \Delta f / 2 m$. As $\Delta f$ is small, the rotation speed is very slow. The slow rotation can clearly be seen in the infrared images, as shown in Fig. 22. It costs $283.6 \mathrm{~s}$ for the nodes to rotate $60^{\circ}$, and the angular velocity is $0.21^{\circ} / \mathrm{s}$.

As thermocouples measure the oscillations at fixed points, which periodically experience the wave trough and the wave peak due to the slow rotation of the standing wave. Therefore, the thermocouples obtain an oscillation with a modulation period of $2 / \Delta f$. This is also called the beat phenomenon, expressed as

$$
T=A \cos (2 \pi \Delta f / 2 t) \cos \left(\frac{2 \pi f_{c}+2 \pi f_{a}}{2} t\right) .
$$

Figure 23 shows the oscillation signals of 6 thermocouples in the experiment with $V r=0.855$. Modulations of amplitude are found at the beginning and the end of the experiment. At the beginning, the long period modulation is caused by the standing wave with $m=4$ and counterpropagating waves with $m=3$ and 4 , shown in
Fig. 18. The modulation disappears after the transition to the anticlockwise traveling wave $(m=3)$. Near the end of experiment, the modulation recurs because of the pattern transition to the standing wave, in which the phase differences of the signal are $0^{\circ}$ or $180^{\circ}$. The modulation period is $270 \mathrm{~s}$, which is the duration for the $\mathrm{m}=3$ pattern rotating in $60^{\circ}$. Therefore, we infer that the slow rotation velocity is $2 \pi \Delta f /(2 \times 3)=0.22^{\circ} / \mathrm{s}$. This is in good agreement with the observation from the infrared camera, which is $0.21^{\circ}$ s. According to the modulation period, we calculate the frequency difference between the counterpropagating waves, which is $0.0037 \mathrm{~Hz}$.

\section{E. Benjamin-Feir instability}

Usually, the petals of regular waves rotate in a constant speed and keep equal gaps with each other. However, we have found a novel pattern whose petal speed is quasi-periodically varied. It

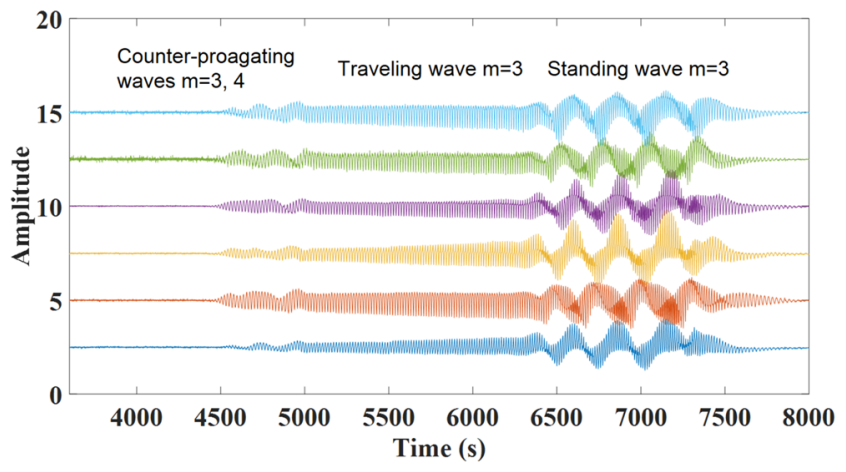

FIG. 23. Detrend oscillation signals by thermocouples. 
occurs in the experiment with $V r=1.045$ when the temperature difference reaches $29.94{ }^{\circ} \mathrm{C}$, which is 1.93 times higher than the critical condition. The wave pattern is shown in Fig. 24. We mark the three petals with Arabic numbers 1, 2, and 3, respectively. It seems that the petals chase each other in the azimuthal direction. First, when $t=0 \mathrm{~s}$, we notice that gaps between the petals are unequal, and there is a large "cavity" between petal 1 and petal 3. At this moment, petal 1 propagates forward with a higher speed and quickly occupies the "cavity." Simultaneously, a new "cavity" is formed between petal 1 and petal 2. Similarly, petal 2 accelerates the rotation and quickly occupies the "cavity." Then, petal 3 occupies the "cavity" ahead, and the new "cavity" is formed between petal 1 and petal 3 at $t=32 \mathrm{~s}$. This is a quasi-periodic pattern dynamics, in which the morphology seldom repeats.

Figure 25 shows the spatial-temporal evolution diagram of the transition from the traveling wave to the novel pattern. At the beginning, the strips are straightly oblique downward, which is a clockwise traveling wave with $m=3$. The straight strips gradually evolve to zig-zag strips which are correlated with the novel pattern. Since the strip inclination stands for the traveling speed, it can be inferred that zig-zag strips stand for sinusoidal variations in velocities of the petals.

Unequal gaps between the petals in Fig. 24 imply that there are other wavenumbers besides $m=3$. To explore the wavenumber selection, we perform a Fourier transformation on the circumferential temperature distribution. The spectra of the traveling wave and this novel pattern are shown in Figs. 26(a) and 26(b), respectively. A single wavenumber $m=3$ is found in the traveling wave, but multiple peaks are found at $m=1,2,3$, and 4 in the novel pattern. This means that there a large band of waves occurring in the novel pattern. Therefore, the formula that only takes a single clockwise/anticlockwise wave into account is not appropriate, and the mixed waves of $m=1,2,3$, and 4 should be considered.

The mixed waves have a side band instability, which is called the Benjamin-Feir instability. It means that the wavenumbers $m-\Delta m$ and $m+\Delta m$ are growing with the fundamental wave, corresponding to $m=2$ and 4 . As we know, the wave frequency increases

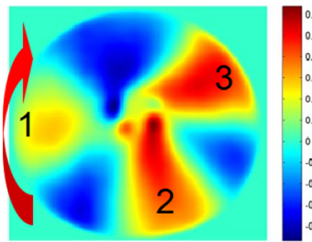

(a1) $t=0 \mathrm{~s}$

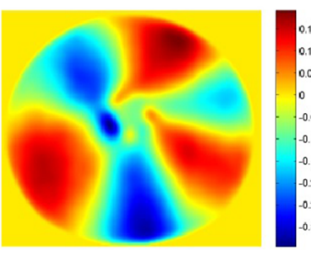

(b1) $t=13.3 \mathrm{~s}$

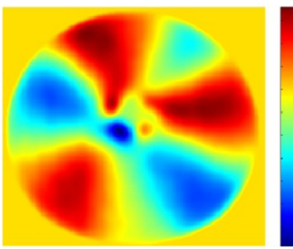

(c1) $t=26.7 \mathrm{~s}$

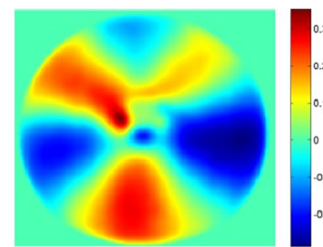

(d1) $t=40.0 \mathrm{~s}$

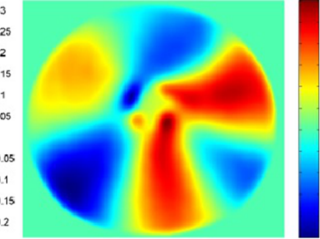

(a2) $t=2.7 \mathrm{~s}$

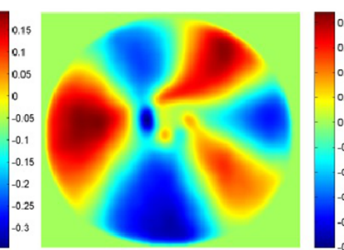

(b2) $t=16.0 \mathrm{~s}$

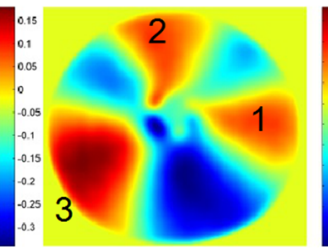

(c2) $t=29.3 \mathrm{~s}$

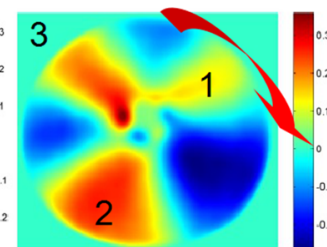

(d2) $t=42.7 \mathrm{~s}$

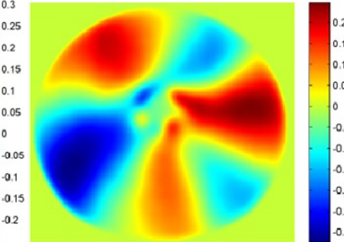

(a3) $t=5.3 \mathrm{~s}$

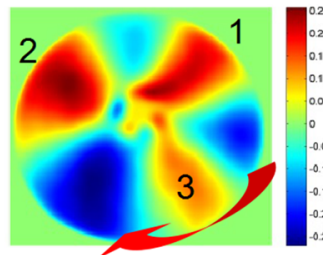

(b3) $t=18.7 \mathrm{~s}$

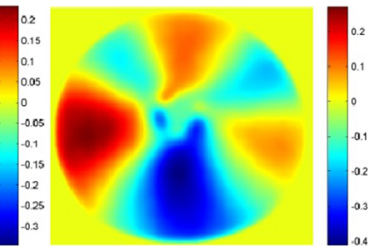

(c3) $t=32.0 \mathrm{~s}$

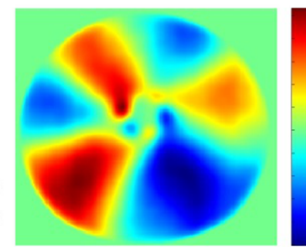

(d3) $t=45.3 \mathrm{~s}$

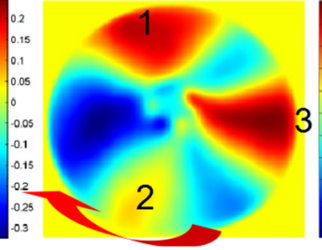

(a4) $t=8.0 \mathrm{~s}$

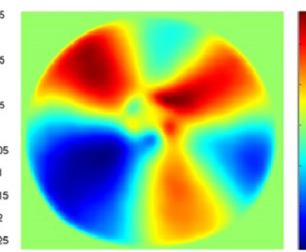

(b4) $t=21.3 \mathrm{~s}$

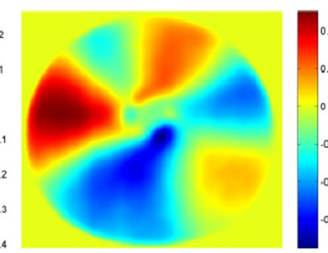

(c4) $t=34.7 \mathrm{~s}$

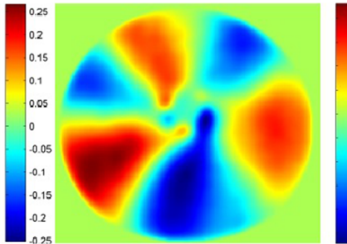

(d4) $t=48.0 \mathrm{~s}$

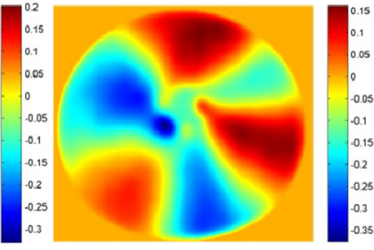

(a5) $t=10.7 \mathrm{~s}$

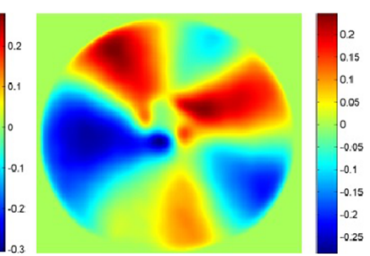

(b5) $t=24.0 \mathrm{~s}$

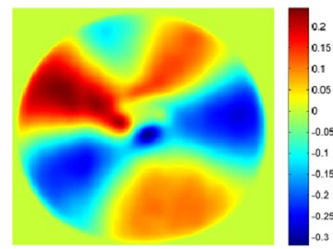

(c5) $t=37.3 \mathrm{~s}$

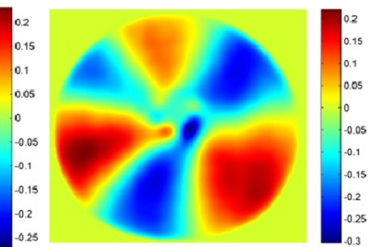

(d5) $t=50.7 \mathrm{~s}$

FIG. 24. Images of the novel pattern in sequence. 


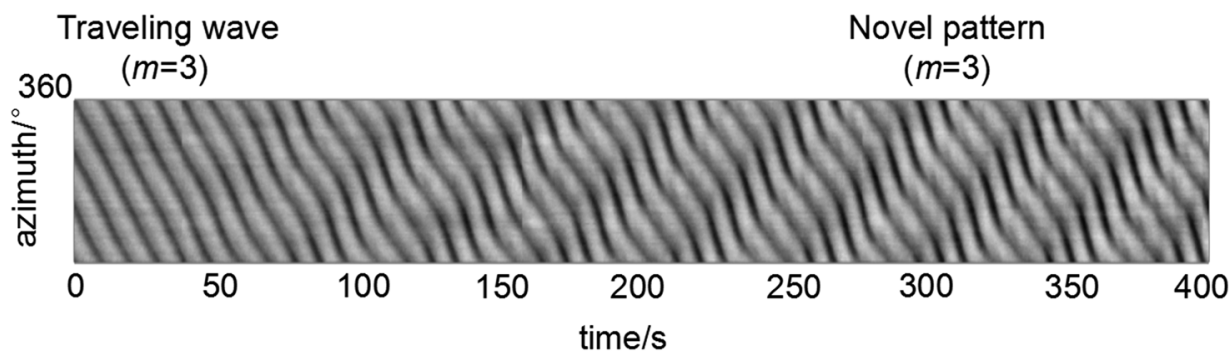

FIG. 25. The spatial-temporal evolution of the novel pattern.

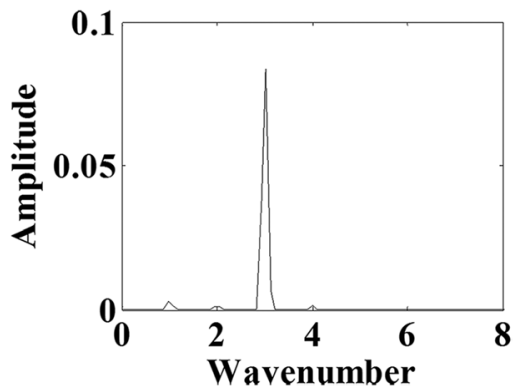

(a)

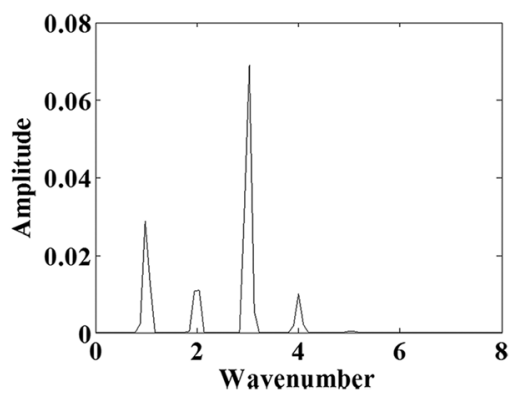

(b)
FIG. 26. Fourier spectra of circumferential temperature distribution. (a) The traveling wave. (b) The novel pattern. as the wavenumber $m$ decreases from 4 to 3 . We assume that the frequencies of waves with $m=2$ and $m=4$ are $f+\Delta f$ and $f-\Delta f$, respectively. The mathematical expression of the Benjamin-Feir instability is assumed as follows:

$$
\begin{aligned}
\mathrm{F}(\theta, r)= & f(r)\left[A_{2} \cos (2 \theta+2 \pi(f+\Delta f) t)+A_{3} \cos (3 \theta+2 \pi f t)\right. \\
& \left.+A_{4} \cos (4 \theta+2 \pi(f-\Delta f) t)\right] .
\end{aligned}
$$

The formula is verified by the space experiment in the following step. First, we determine the parameters $\Delta f$ and $A_{i}(i=1,2$, and 3$)$ by the data of thermocouples (Fig. 27) and then compare the spatialtemporal evolution of Eq. (17) with infrared images (Fig. 25). If the model determined by the thermocouple signal explains the spatial evolution of infrared images, the validity of the model can be verified. In Fig. 27, stable oscillations are found in the traveling wave and modulations (beat phenomena) are found in the novel oscillation. The spectra of the traveling wave and the novel wave are shown in Fig. 28. Two fundamental frequencies $f$ and $\Delta f$ are found in the spectra of the novel pattern, and they are $0.05341 \mathrm{~Hz}$ and $0.01144 \mathrm{~Hz}$, respectively. Other peaks can be calculated by linear combinations of $f$ and $\Delta f$, such as $f-\Delta f, f+\Delta f, 2 f, 2 f-\Delta f$, and $2 f+\Delta f$.

The model assumes that the frequencies of waves with $m=2$, 3 , and 4 are $f+\Delta f, f$, and $f-\Delta f$, respectively. The amplitudes, $A_{2}$, $A_{3}$, and $A_{4}$, are obtained from the square roots of power spectral densities of $f+\Delta f$, $f$, and $f-\Delta f$. We obtain $A_{2}=1.53, A_{3}=6.12$, and $A_{4}=2.79$. As the phases of $m=2$ wave and $m=4$ wave are different in $\pi$, we set $A_{2}=1.53$ and $A_{4}=-2.79$, which is equivalent to $A_{2}=-1.53$ and $\mathrm{A}_{4}=2.79$ by time shifting translation. After the amplitudes and frequencies are determined, the spatial-temporal evolution is obtained by Eq. (17), as shown in Fig. 29. The model generates zig-zag strips, which are in good agreement with the space experiments. Therefore, the chasing phenomenon of the novel wave is caused by the mixed waves of $m=2,3$, and 4 , which is well described by Eq. (17).

Furthermore, if we do not neglect the energy of the frequency $\Delta f$, an anticlockwise wave with $m=1$ should be added to the mathematical model, which gives

$$
\begin{aligned}
\mathrm{F}(\theta, r)= & f(r)\left[A_{1} \cos (\theta-2 \pi \Delta f t)+A_{2} \cos (2 \theta+2 \pi(f+\Delta f) t)\right. \\
& \left.+A_{3} \cos (3 \theta+2 \pi f t)+A_{4} \cos (4 \theta+2 \pi(f-\Delta f) t)\right] .
\end{aligned}
$$

The amplitude $A_{1}$ is 4.49 given by the spectra, and the spatialtemporal evolution is shown in Fig. 30. Different from the oblique downward strips in Fig. 29, bright-dark upward strips are found. The downward oblique strips with a smaller slope are in the bright area,

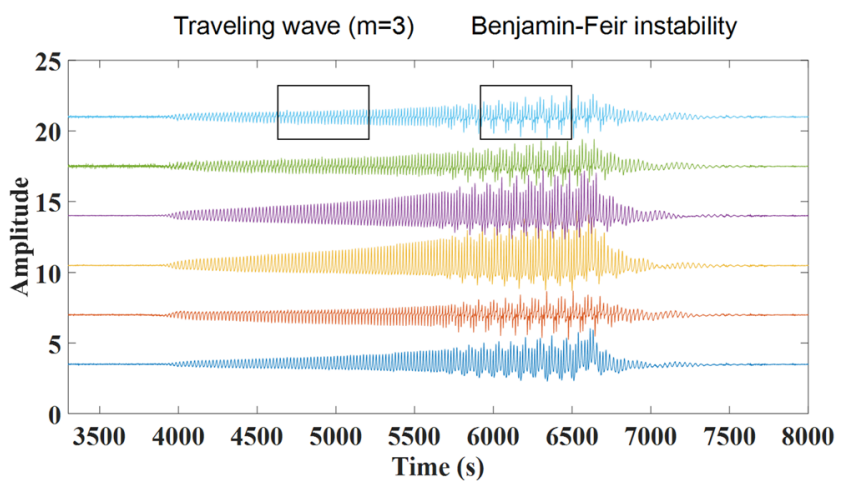

FIG. 27. The temperature oscillation of the Benjamin-Feir instability. 


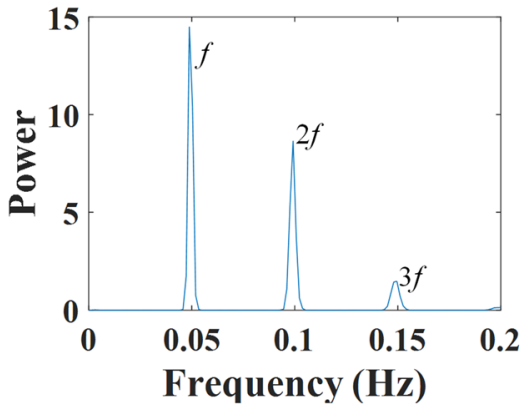

(a)

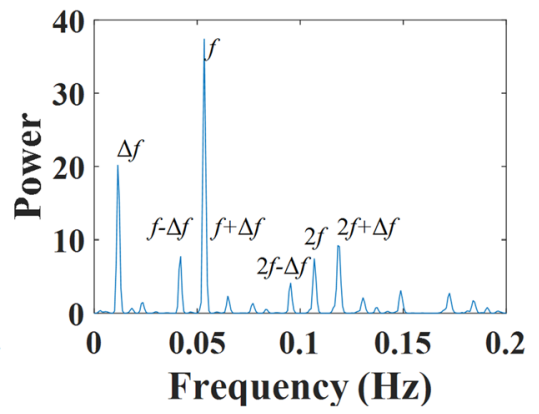

(b)
FIG. 28. The spectrum analysis of the oscillation. (a) The traveling wave. (b) Benjamin-Feir instability.
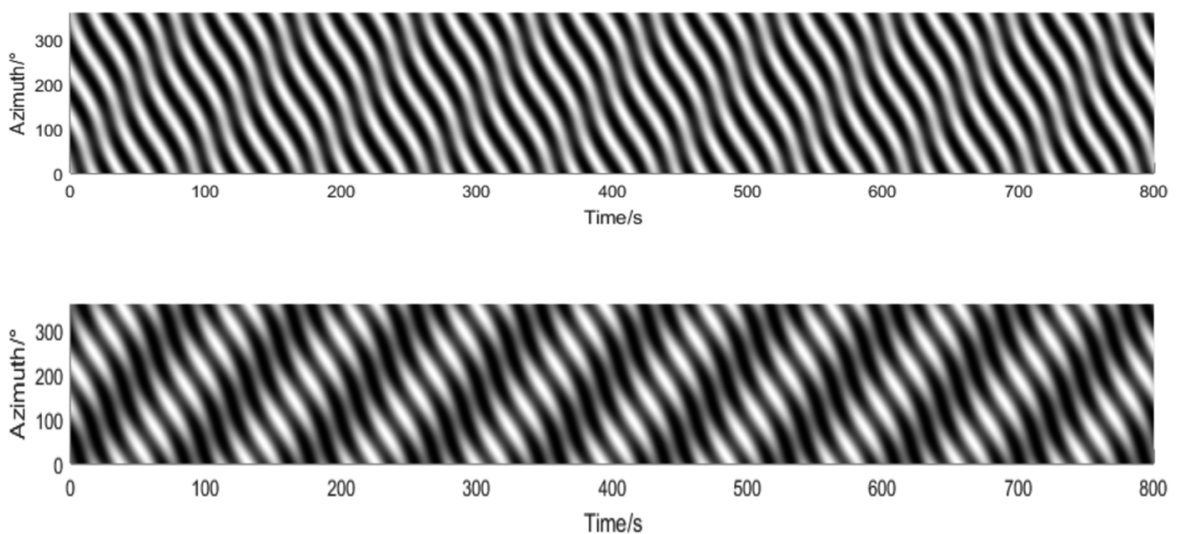

FIG. 29. The spatial-temporal evolution of the superposition of waves with $m=2$, 3 , and 4 . and the strips with a larger slope are in the dark area. It means that the faster propagating waves have smaller amplitudes and slower propagating waves have larger amplitudes. This is in agreement with experiments. As shown in Fig. 24, the faster petals, such as petal 1 in Fig. 24(a1), petal 2 in Fig. 24(a4), and petal 3 in Fig. 24(b3), present weak amplitude.

\section{CONCLUSIONS}

Surface configuration, defined by the volume ratio, has significant effects on the basic flow state and thermocapillary oscillation in a geometrically fixed annular open cavity. The configurations are classified into 3 regimes. When $V r<0.77$ (zone 1), the free surface is a concave meniscus and the inner contact line is located beneath the top of the heating column. When $0.77<V r<1$ (zone 2), though the surface is concave, the contact lines are pinned by the designed wedges on the top. When $V r>1$ (zone 3), the inner contact line passes the wedge and attaches to the top column, forming a convex liquid surface. The basic flow is a single vortex structure in most of the experiments. But in the experiments at very small volume ratios, for example, $V r=0.363$ and $V r=0.423$, we also find the double vortex structure. The thickness of the thermal boundary layer in the basic flow decreases with the increase in temperature difference. The boundary thickness approximates to a minimum and the basic flow saturates to a nonlinear configuration, when the temperature difference is higher than $10{ }^{\circ} \mathrm{C}$. In the nonlinear basic flow, instability makes the thermocapillary convection transit from the steady flow to the oscillatory flow. The critical temperature difference of the oscillation shows a significant volume ratio effect, by which the critical curve can be divided into two branches. The left branch is for $V r<0.65$, and its critical temperature difference increases with the increase in volume ratio. It performs as a local irregular oscillation in this region. The right branch is for $V r>0.65$, and the critical temperature difference decreases with the increase in volume ratio. The oscillations in the right branch are regular azimuthal waves with the wavenumber $m=3$ or 4 . The azimuthal waves evolve from the standing wave to the traveling wave. The wave modes and the transition process are affected by the volume ratio. The wavenumber is 4 in zone 1 , and the wavenumber is 3 in zone 3 . However, in zone 2, there is competition between wavenumbers $m=3$ and $m=4$. As the temperature difference increases, the mode transition from $m=4$ to $m=3$ is found. During this transition, the counterpropagate waves with $m=3$ and 4 are also found, and they finally develop into a steady traveling wave with $m=3$. The transition from the traveling wave to the standing wave and the Benjamin-Feir instability are found at a high temperature difference.

\section{ACKNOWLEDGMENTS}

The authors gratefully acknowledge Pu Zhang, Zhiyi Han, and Mingzhu $\mathrm{Hu}$ for participating in the monitoring of the space experiments; Li Zhang and Di Zhang for conducting preliminary ground experiments of thermocapillary convection in the annular pool; and YongLi Yin, Jingsong Yang, Sifang Zhao, and Shuangbo Li from 
the Astronaut Center of China for participating in the research and development of the Space experimental equipment.

This work was supported by the Strategic Priority Research Program on Space Science of Chinese Academy of SciencesSJ-10 Recoverable Scientific Experiment Satellite (Grant Nos. XDA04020405 and XDA04020202-05) - and by the National Natural Science Foundation of China (Grant No. U1738116).

\section{REFERENCES}

${ }^{1}$ H. Benard, "Les tourbillons cellulaires dans une nappe liquide," Rev. Gen. Sci. Pures Appl. 11, 1261 (1900).

${ }^{2}$ S. H. Davis, "Thermocapillary instabilities," Annu. Rev. Fluid Mech. 19(1), 403-435 (1987).

${ }^{3}$ M. F. Schatz and G. P. Neitzel, "Experiments on thermocapillary instabilities," Annu. Rev. Fluid. Mech. 33(1), 93-127 (2001).

${ }^{4} \mathrm{M}$. Jurisch and W. Löser, "Analysis of periodic non-rotational W striations in Mo single crystals due to nonsteady thermocapillary convection," J. Cryst. Growth 102(1), 214-222 (1990).

${ }^{5}$ D. Schwabe and A. Scharmann, "Some evidence for the existence and magnitude of a critical Marangoni number for the onset of oscillatory flow in crystal growth melts," J. Cryst. Growth 46(1), 125-131 (1979).

${ }^{6}$ O. M. F. R. S. Lord Rayleigh, "LIX. On convection currents in a horizontal layer of fluid, when the higher temperature is on the under side," Philos. Mag. 32(192), 529-546 (1916).

${ }^{7}$ J. R. A. Pearson, “On convection cells induced by surface tension," J. Fluid Mech. 4(5), 489-500 (2006).

${ }^{8}$ E. L. Koschmieder and M. I. Biggerstaff, "Onset of surface-tension-driven Benard convection," J. Fluid Mech. 167(-1), 49-64 (1986).

${ }^{9} \mathrm{~K}$. Nitschke, "Secondary instability in surface-tension-driven Benard convection," Phys. Rev. E 52(6), R5772-R5775 (1995).

${ }^{10}$ A. A. Nepomnyashchy and I. B. Simanovskii, "Influence of buoyancy on thermocapillary oscillations in a two-layer system," Phys. Rev. E 68(2), 026301 (2003).

${ }^{11}$ L. Lu-Jun, D. Li, H. Liang et al., "Experimental investigation of influence of interfacial tension on convection of two-layer immiscible liquid," Chin. Phys. Lett. 25(5), 1734 (2008).

${ }^{12}$ M. K. Smith and S. H. Davis, "Instabilities of dynamic thermocapillary liquid layers. I Convective instabilities," J. Fluid Mech. 132(-1), 119 (1983).

${ }^{13} \mathrm{~J}$. Burguete, N. Mukolobwiez, F. Daviaud et al., "Buoyant-thermocapillary instabilities in extended liquid layers subjected to a horizontal temperature gradient," Phys. Fluids 13(10), 2773-2787 (2001).

${ }^{14}$ D. Schwabe, U. Möller, J. Schneider et al., "Instabilities of shallow dynamic thermocapillary liquid layers," Phys. Fluids A 4(11), 2368-2381 (1992).

${ }^{15}$ A. B. Ezersky, A. Garcimartín, J. Burguete et al., "Hydrothermal waves in Marangoni convection in a cylindrical container," Phys. Rev. E 47(2), 1126 (1993).

${ }^{16}$ J.-J. Xu and S. H. Davis, "Convective thermocapillary instabilities in liquid bridges," Phys. Fluids 27(5), 1102-1107 (1984).

${ }^{17}$ D. Schwabe, A. Scharmann, F. Preisser et al., "Experiments on surface tension driven flow in floating zone melting," J. Cryst. Growth 43(3), 305-312 (1978).

${ }^{18}$ F. Preisser, D. Schwabe, and A. Scharmann, "Steady and oscillatory thermocapillary convection in liquid columns with free cylindrical surface," J. Fluid Mech. 126(126), 545-567 (1983).

${ }^{19}$ J. Wang, D. Wu, L. Duan, and Q. Kang, "Ground experiment on the instability of buoyant-thermocapillary convection in large-scale liquid bridge with large Prandtl number," Int. J. Heat Mass Transfer 108, 2107-2119 (2017).

${ }^{20} \mathrm{D}$. Schwabe, "Hydrothermal waves in a liquid bridge with aspect ratio near the Rayleigh limit under microgravity," Phys. Fluids 17(11), 112104 (2005).
${ }^{21}$ H. Kawamura, K. Nishino, S. Matsumoto et al., "Report on microgravity experiments of Marangoni convection aboard international space station," J. Heat Transfer 134(3), 031005 (2012).

${ }^{22}$ K. Nishino, T. Yano, H. Kawamura, S. Matsumoto, I. Ueno, and M. K. Ermakov, "Instability of thermocapillary convection in long liquid bridges of high Prandtl number fluids in microgravity," J. Cryst. Growth 420, 57-63 (2015).

${ }^{23}$ T. Yano, K. Nishino, S. Matsumoto et al., "Report on microgravity experiments of dynamic surface deformation effects on Marangoni instability in high-Prandtlnumber liquid bridges," Microgravity Sci. Technol. 30, 599 (2018).

${ }^{24} \mathrm{M}$. Levenstam, "Hydrodynamical instabilities of thermocapillary flow in a halfzone," J. Fluid Mech. 297(-1), 357-372 (1995).

${ }^{25}$ K. Motegi, K. Fujimura, and I. Ueno, "Floquet analysis of spatially periodic thermocapillary convection in a low-Prandtl-number liquid bridge," Phys. Fluids 29(7), 074104 (2017).

${ }^{26}$ C. D. Brandle, "Simulation of fluid flow in $\mathrm{Gd}_{3} \mathrm{Ga}_{5} \mathrm{O}_{12}$ melts," J. Cryst. Growth 42, 400-404 (1977).

${ }^{27}$ D. C. Miller and T. L. Pernell, "The temperature distribution in a simulated garnet Czochralski melt," J. Cryst. Growth 53(3), 523-529 (1981).

${ }^{28}$ T. Azami, S. Nakamura, M. Eguchi et al., "The role of surface-tension-driven flow in the formation of a surface pattern on a Czochralski silicon melt," J. Cryst. Growth 233(1), 99-107 (2001).

${ }^{29} \mathrm{~N}$. Garnier, A. Chiffaudel, and F. Daviaud, "Hydrothermal waves in a disk of fluid," in Dynamics of Spatio-Temporal Cellular Structures (Springer, New York, 2006), pp. 147-161.

${ }^{30}$ Y. R. Li, L. Zhang, L. Zhang et al., "Experimental study on Prandtl number dependence of thermocapillary-buoyancy convection in Czochralski configuration with different depths," Int. J. Therm. Sci. 130, 168-182 (2018).

${ }^{31}$ J. J. Yu, D. F. Ruan, Y. R. Li et al., "Experimental study on thermocapillary convection of binary mixture in a shallow annular pool with radial temperature gradient," Exp. Therm. Fluid Sci. 61, 79-86 (2015).

${ }^{32}$ T. Shen, C. M. Wu, L. Zhang et al., "Experimental investigation on effects of crystal and crucible rotation on thermal convection in a model Czochralski configuration," J. Cryst. Growth 438, 55 (2016).

${ }^{33}$ T. Shen, C. M. Wu, and Y. R. Li, "Experimental investigation on the effect of crystal and crucible rotation on thermocapillary convection in a Czochralski configuration," Int. J. Therm. Sci. 104, 20-28 (2016).

${ }^{34} \mathrm{Q}$. Kang, L. Duan, L. Zhang et al., "Thermocapillary convection experiment facility of an open cylindrical annuli for SJ-10 satellite," Microgravity Sci. Technol. 28(2), 123-132 (2016).

${ }^{35} \mathrm{D}$. Longsheng, D. Li, J. Huan et al., "Oscillation transition routes of buoyantthermocapillary convection in annular liquid layers," Microgravity Sci. Technol. 30, 865 (2018).

${ }^{36}$ D. Schwabe and S. Benz, "Thermocapillary flow instabilities in an annulus under microgravity-Results of the experiment magia," Adv. Space Res. 29(4), 629-638 (2002).

${ }^{37}$ D. Schwabe, A. Zebib, and B. C. Sim, "Oscillatory thermocapillary convection in open cylindrical annuli. Part 1. Experiments under microgravity," J. Fluid Mech. 491(491), 239-258 (2003).

${ }^{38}$ Y. Kamotani, A. Chang, and S. Ostrach, "Effects of heating mode on steady axisymmetric thermocapillary flows in microgravity," J. Heat Transfer 118, 191 (1994).

${ }^{39}$ Y. Kamotani, S. Ostrach, and J. Masud, "Microgravity experiments and analysis of oscillatory thermocapillary flows in cylindrical containers," J. Fluid Mech. 410, 211-233 (2000)

${ }^{40}$ Y. Kamotani, S. Ostrach, and A. Pline, "Some temperature field results from the thermocapillary flow experiment aboard USML-2 spacelab," Adv. Space Res. 22, 1189-1195 (1998).

${ }^{41}$ B. C. Sim, A. Zebib, and D. Schwabe, "Oscillatory thermocapillary convection in open cylindrical annuli. Part 2. Simulations,” J. Fluid Mech. 491, 259-274 (2003).

${ }^{42}$ Y. R. Li, L. Peng, Y. Akiyama et al., "Three-dimensional numerical simulation of thermocapillary flow of moderate Prandtl number fluid in an annular pool," J. Cryst. Growth 259(4), 374-387 (2003). 
${ }^{43}$ B. C. Sim and A. Zebib, "Effect of free surface heat loss and rotation on transition to oscillatory thermocapillary convection," Phys. Fluids 14(1), 225 (2002).

${ }^{44}$ B. C. Sim and A. Zebib, "Thermocapillary convection with undeformable curved surfaces in open cylinders," Int. J. Heat Mass Transfer 45(25), 4983-4994 (2002).

${ }^{45}$ R. Lavalley, G. Amberg, and H. Alfredsson, "Experimental and numerical investigation of nonlinear thermocapillary oscillations in an annular geometry," Eur. J. Mech.: B/Fluids 20, 771 (2001).
${ }^{46}$ W. R. Hu, J. F. Zhao, M. Long et al., "Space program SJ-10 of microgravity research," Microgravity Sci. Technol. 26, 159 (2014).

${ }^{47}$ B. C. Sim, W. S. Kim, and A. Zebib, "Axisymmetric thermocapillary convection in open cylindrical annuli with deforming interfaces," Int. J. Heat Mass Transfer 47(24), 5365-5373 (2004).

${ }^{48}$ W. R. Hu, J. Z. Shu, R. Zhou et al., "Influence of liquid bridge volume on the onset of oscillation in floating zone convection I. Experiments," J. Cryst. Growth 142(3-4), 379-384 (1994). 